\title{
Locomotion Optimization and Manipulation Planning of a Tetrahedron-Based Mobile Mechanism with Binary Control
}

\author{
Ran Liư ${ }^{1}$, Yan-An Yao ${ }^{1 *}$, Wan Ding ${ }^{2}$ and Xiao-Ping Liu ${ }^{1,3}$
}

\begin{abstract}
Locomotion and manipulation optimization is essential for the performance of tetrahedron-based mobile mechanism. Most of current optimization methods are constrained to the continuous actuated system with limited degree of freedom (DOF), which is infeasible to the optimization of binary control multi-DOF system. A novel optimization method using for the locomotion and manipulation of an 18 DOFs tetrahedron-based mechanism called 5-TET is proposed. The optimization objective is to realize the required locomotion by executing the least number of struts. Binary control strategy is adopted, and forward kinematic and tipping dynamic analyses are performed, respectively. Based on a developed genetic algorithm (GA), the optimal number of alternative struts between two adjacent steps is obtained as 5. Finally, a potential manipulation function is proposed, and the energy consumption comparison between optimal 5-TET and the traditional wheeled robot is carried out. The presented locomotion optimization and manipulation planning enrich the research of tetrahedron-based mechanisms and provide the instruction to the successive locomotion and operation planning of multi-DOF mechanisms.
\end{abstract}

Keywords: Tetrahedron-based mobile mechanism, Binary control, GA, Locomotion optimization, Manipulation planning

\section{Introduction}

Terrestrial mobile robots mainly include wheeled, tracked, legged, hybrid, snake-like, and spherical robots $[1,2]$, which have different adaptability and locomotion modes. Different from conventional robots that actuated by motors inside to hold a constant shape, some mechanisms realize locomotion and manipulation functions by shifting shapes with the change of the linkages [3]. In recent years, some linkage structures constructed by basic or special geometry configurations, such as Euclidean polyhedron, have been proposed. Conformable tetrahedrons are the simplest space-filling form in the same way triangles are the simplest plane-filling facets [4]. The

\footnotetext{
*Correspondence: yayao@bjtu.edu.cn

${ }^{1}$ School of Mechanical, Electronic and Control Engineering, Beijing Jiaotong University, Beijing 100044, China

Full list of author information is available at the end of the article
}

tetrahedron-based mobile mechanism has been commonly explored.

Tetrahedron-based mobile mechanism is a class of hyper-redundant robots, which has multiple DOFs and kinematic redundancy [5]. Different from wheeled, tracked, and legged robots, hyper-redundant mechanisms arise from internally induced deforming. Since the advantage of fault tolerance [6], it is superior for operation in highly constrained environments, for instance, in uneven terrain with some simple operation tasks, such as nuclear reactor cores, toxic abandoned factories, and many other conceivable environments [7].

Several tetrahedron-based robots have been designed, and the emphasis mainly on building novel structures to produce feasible gait patterns. Based on the mathematical models of 4-TET, 8-TET, 12-TET Walkers and tetrahedron worm built by Abrahantes et al. [4, 8], the choreographed gaits of these robots were designed 
according to the geometric relationships of the struts. For a novel tensegrity duct robot constructed with two linked tetrahedrons $[9,10]$, the main focus is also on the design of climbing gaits. Through the structure design and kinematic analysis of a steering crawling tetrahedron robot which links a pushing element on one of its four nodes [11], the slope crawling gait was presented. However, the research on successive path planning of such linkage-based mobile mechanism is relatively rare. And its successive motion much depends on complex control system. A light source tracking 1-TET designed by $\mathrm{Yu}$ and Nagpal [12] realize its rolling motion by control the self-adapting system contains amounts of sensors. And a spine tensegrity robot simulated its successive rolling by using central pattern generators (CPGs) [13].

Since the large redundancy of multi-DOF robots, the key issue of the realization of successive locomotion lies on the inverse kinematics, which is a nonlinear problem that has multiple solutions [14]. A two-linked tetrahedron robot adopted force density method to solve the inverse kinematics [9] for the minimum of elastic potential energy contained. In Ref. [15], a GA was used to solve the inverse kinematics of a redundant robot, which mainly focuses on finding the best solution among multiple solutions with the objective of minimum joint displacements.

Tetrahedron-based robots are always over-constrained, which requires to embed large extension ratio actuators to acquire large deformation and high mobility [16]. Since there are few actuators meet this special requirement, researchers have explored using ordinary extension ratio actuators to design modular reconfigurable robotic systems with high environmental adaptability $[17,18]$. One of the modular reconfigurable robots called Tetrobot [5] that can be reconstructed as tetrahedron module, octahedral module, six-legged walker, and tetrahedron-based manipulator. Each of these four constructions can meet the respectively given tasks. Another modular reconfigurable robot [12] has three reconfigurable constructions, including an adaptive gripper, a modular tetrahedron robot, and a modular hexahedron robot. However, the three constructions can only meet its corresponding task. If there were only one construction that could meet more than one task, it would be very desirable [19].

In previous work, we reported a closed-loop mechanism consisted of two tetrahedron units with two DOFs that could realize rolling locomotion on the ground [20]. And a pneumatic driving tetrahedron mechanism with multi-DOF was proposed [21]. In this paper, we focused mainly on the locomotion optimization and manipulation planning of the tetrahedron-based mobile mechanism constructed as 5-TET. A binary control strategy [22, 23] using binary actuators is adopted to simplify the control system. A GA based on binary codes is developed to optimize the locomotion of 5 -TET by altering minimum actuators, which further simplifies the control of the mechanism. Double control simplification provides a reference for multi-DOF inputs combination strategy.

This paper is organized as follows. The structure and locomotion of 5-TET are described in Section 2. Section 3 illustrates the optimal locomotion based on kinematic and dynamic analyses. Section 4 presents a potential operation function of 5-TET. Energy consumption is analyzed Section 5. And the optimal locomotion paths and manipulation gaits are simulated Section 6.

\section{Structure and Locomotion Description}

\subsection{Structure Description}

Geometrically, 5-TET is comprised of 18 identical struts and 8 nodes, which is a fully symmetrical spatial geometry with isotropy feature. Mechanically, substituting prismatic joints for each of the 18 struts and using spherical joints connect the struts end to end, which make up the 5-TET mobile mechanism with 18 DOFs, corresponding to each of the 18 prismatic actuators (see in Figure 1).

\subsection{Binary Control and System Implementation}

Binary control is one of the control concept applied to multi-DOF mechanisms [24]. In this concept, tens or hundreds of binary actuators are embedded in a structure, which is analogous to the digital computer replacing the analog computer. These digital mechanisms can perform precise, discrete motions without the need of sensing, complex electronics or feedbacks, so the control elements of such devices are simple [25]. Since the binary control only has signals of 0 and 1 that correspond to off and on, the control operation of such devices is also simple [23].

Taking the advantages of quick response, large strength-weight ratio, and lower control complexity,

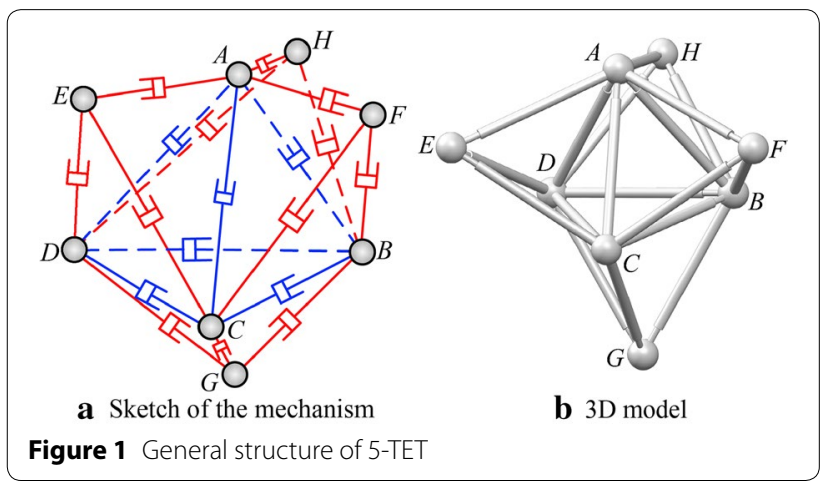


pneumatic cylinders are selected as the binary actuators for 5-TET. Pneumatic cylinder has only ' $\mathrm{min} / \mathrm{max}$ ' length, which correspond to the binary codes of ' $0 / 1$ '. However, since the binary behavior, the feasible configurations of binary mechanisms would decrease. To remedy this limitation, it is preferred to assemble several modules in the architecture to realize a quasi-continuous mobility [26].

For mobile robot, the integration design concept is a good choice. Since the embedding of pneumatic elements, the implementation of 5-TET calls for the pneumatic power supply, which is a dominant bottleneck in practical of pneumatic mobile applications. Many researchers have been studied on this problem, and new advances in portable pneumatic power source are presented [27-29]. For example, a portable pneumatic supply called Dry Ice Power Cell, which could provide sustaining $0.42 \mathrm{MPa}$ gas for about $218 \mathrm{NL}$ with portable size [29]. The achievements from these researches can be used directly to equip 5-TET to realize mobile function, so do other elements.

According to the parameters of existing pneumatic actuators with the consideration of the global dimension of 5-TET, choose the specifications of the pneumatic cylinders in Table 1. Each actuator weighs $0.5 \mathrm{~kg}$. The two extreme states are $360 \mathrm{~mm}$ and $460 \mathrm{~mm}$. Here, the stroke is chosen as small as possible to verify the 5 -TET is independent of large extension ratio. Accordingly, the nodes are designed with the radius of $30 \mathrm{~mm}$, whose mass center locates in its geometry center and equals to $0.3 \mathrm{~kg}$.

The implemental system of integration design concept mainly contains the 5-TET mechanism, the pneumatic system (including a pneumatic power supply, 18 electric solenoid valves and some pneumatic tube), a control unit with battery, a wireless unit and a remote computer. 5 -TET is an executive mechanism that activated by electronic solenoid valves when the control unit receives the optimal binary codes from the computer by the wireless unit.

For the arrangement of these elements, the portable pneumatic supply would be suspended in the basic tetrahedron A-BCD from nodes $A$ to $D$ by spring with appropriate stiffness that could absorb shock in the rolling.

Table 1 Structure parameters of 5-TET mechanism

\begin{tabular}{ll}
\hline Structure items & Parameters \\
\hline Length range of pneumatic cylinder $/ / \mathrm{mm}$ & $360-460$ \\
Mass of a pneumatic cylinder $m_{\mathrm{L}} / \mathrm{kg}$ & 0.5 \\
Pressure range of pneumatic cylinder $P / \mathrm{MPa}$ & $0.1-0.9$ \\
Radius of a spherical joint $r_{\mathrm{N}} / \mathrm{mm}$ & 30 \\
Mass of a spherical joint $m_{\mathrm{N}} / \mathrm{kg}$ & 0.3 \\
\hline
\end{tabular}

And the valves, control units and other elements are integrated to the other four nodes of $E$ to $H$. However, this leads to the position change of the center of mass (CM). Thus counterweight is used to be added on the nodes to stay the initial CM locates in the center of 5-TET. Then connect the system with pneumatic tube in neat formation. Figure 2 shows the integration design concept of 5-TET.

\subsection{Locomotion Feasibility of 5-TET}

In the condition of ground support, as described in Figure 3, 5-TET remains in three-point support as $\triangle$ DEG. Through controlling the corresponding pneumatic cylinders, 5-TET can roll in three possible directions on condition that the projection of the CM statically or the Zero Moment Point (ZMP) dynamically of the mechanism locates beyond the support area. In Figure 3, 5-TET has three rolling directions with two types of rolling axes from Direction I to III. Obviously Direction I and II have the symmetric motion, which are grouped. Further, with

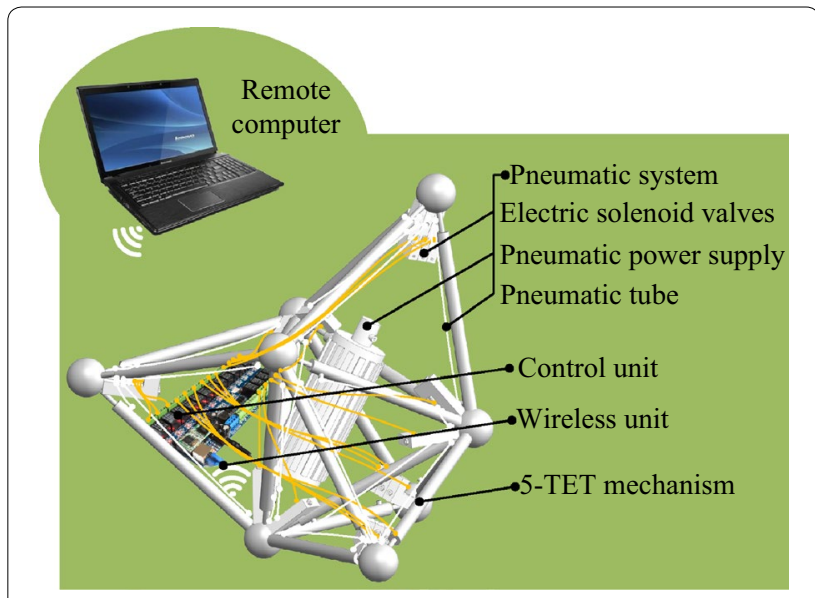

Figure 2 Integration design concept of 5-TET

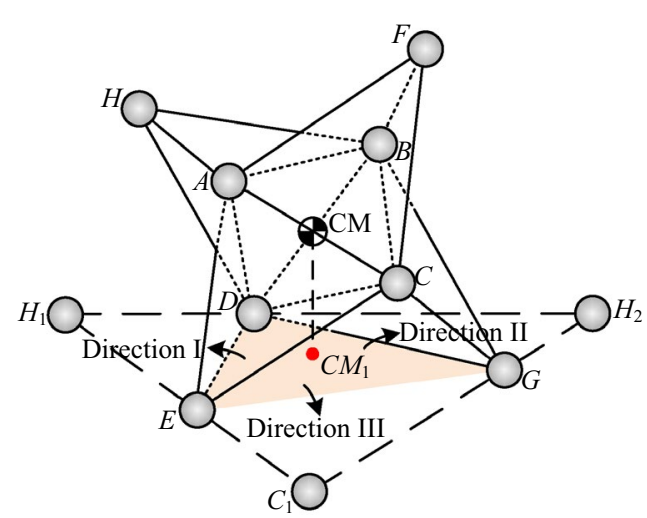

Figure 3 Tipping directions of 5-TET 
successive and logical planning on cooperates of the actuators, 5-TET can realize omnidirectional rolling.

Note that since the mass of telescopic strut is uniform and all the surfaces have the same friction coefficient, the two nodes of the tipping axis bear the same friction. Thus, if the length of tipping axis changes, it would be stretched symmetrically with respect to the center of the pneumatic cylinder. For example, based on the initial state of 5-TET, if the tipping axis is $D E$, and the tipping would happen with the extension of strut DE. Considering that nodes $D$ and $E$ bear the same friction, strut DE will be extended symmetrically with respect to its own center, as described in Figure 4. This feature has a great significance in the locomotion of 5-TET.

For 5-TET, in any static stable state, the support triangle surface is composed of three of the eight nodes. And one of the three nodes is chosen from four nodes of the basic tetrahedron $\mathrm{A}-\mathrm{BCD}$, while the other two are chosen from the four external nodes $E, F, G$ and $H$. But during the moving, the support area of 5-TET is always changing, which deeply varies the kinematic analysis. Fortunately, 5-TET is a fully symmetric configuration that has isotropic feature [30], so that it has identical structures with different support nodes. Using this significant feature, the complexity in kinematic analysis would be reduced.

\section{Motion Gait Optimization and Optimal Path Planning}

For mobile mechanism, it is meaningful to find the optimal input of the required locomotion. To improve the efficiency and accuracy of the optimization, an analytical kinematic model based on geometric relationship rather than coordinate transformation was built. Then based on the tipping dynamic analysis, the motion gaits of a single step and successive locomotion were optimized.

\subsection{Geometric-Based Forward Kinematics}

For tetrahedron-based mechanism, it is complicated and time-consuming to analyze the forward kinematics with coordinate transformation, which directly affects

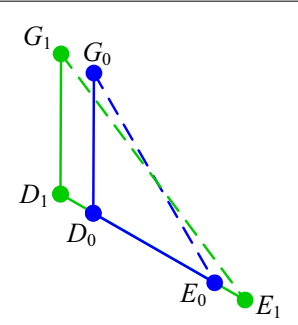

Figure 4 Extension principle of the struts the efficiency of the locomotion optimization. However, based on a fixed coordinate system, the analytical solution could be quickly obtained by the proper use of the geometrical relationship of the mechanism.

For 5-TET, the fixed Cartesian coordinates $O-X Y Z$ is built. See in Figure 5, node $D$ is chosen to be the origin, axis $Y$ is along the direction of vector $D B$, axis $Z$ is perpendicular to the surface of $\triangle \mathrm{BCD}$ just points to the center of tetrahedron $\mathrm{A}-\mathrm{BCD}$, and the $X O Y$ plane is coplanar with $\triangle B C D$. The lengths of all the 18 pneumatic cylinders are expressed as a matrix with 18 lines and 1 column as Length $=[A D, A C, A B, D C, B C, D B, E D, E C$, $E A, G D, G C, G B, H D, H A, H B, F A, F C, F B]^{\mathrm{T}}=\left[a_{1}, b_{1}, c_{1}\right.$, $\left.a_{2}, b_{2}, c_{2}, a_{3}, b_{3}, c_{3}, a_{4}, b_{4}, c_{4}, a_{5}, b_{5}, c_{5}, a_{6}, b_{6}, c_{6}\right]^{\mathrm{T}}$.

In this coordinates, the positions of all nodes of 5-TET could be obtained by geometric calculation. It is obvious that for the basic tetrahedron A-BCD, three out of the four nodes can be positioned easily as follows:

$$
\begin{aligned}
& \underset{\mathbf{B}}{\mathbf{O}} \mathbf{P}=\left({ }_{B}^{O} x,{ }_{B} y,{ }_{B}^{O} z\right)^{\mathrm{T}}=\left(0, c_{2}, 0\right)^{\mathrm{T}}, \\
& { }_{\mathbf{C}}^{\mathrm{O}} \mathbf{P}=\left(\begin{array}{c}
{ }_{C}^{O} \\
C_{C}^{O} y \\
C_{C}^{O} z
\end{array}\right)=\left(\begin{array}{c}
a_{2} \sqrt{1-\left(\frac{a_{2}^{2}+c_{2}^{2}-b_{2}^{2}}{2 a_{2} c_{2}}\right)^{2}} \\
\frac{a_{2}^{2}+c_{2}^{2}-b_{2}^{2}}{2 c_{2}} \\
0
\end{array}\right), \\
& { }_{\mathbf{D}}^{O} \mathbf{P}=\left({ }_{D}^{O} x,{ }_{D}^{O} y, D_{D}^{O} z\right)^{\mathrm{T}}=(0,0,0)^{\mathrm{T}} .
\end{aligned}
$$

The rest is node $A$. According to geometric thinking, a new position analysis method based on the relations between sides and angles is derived to analyze the forward

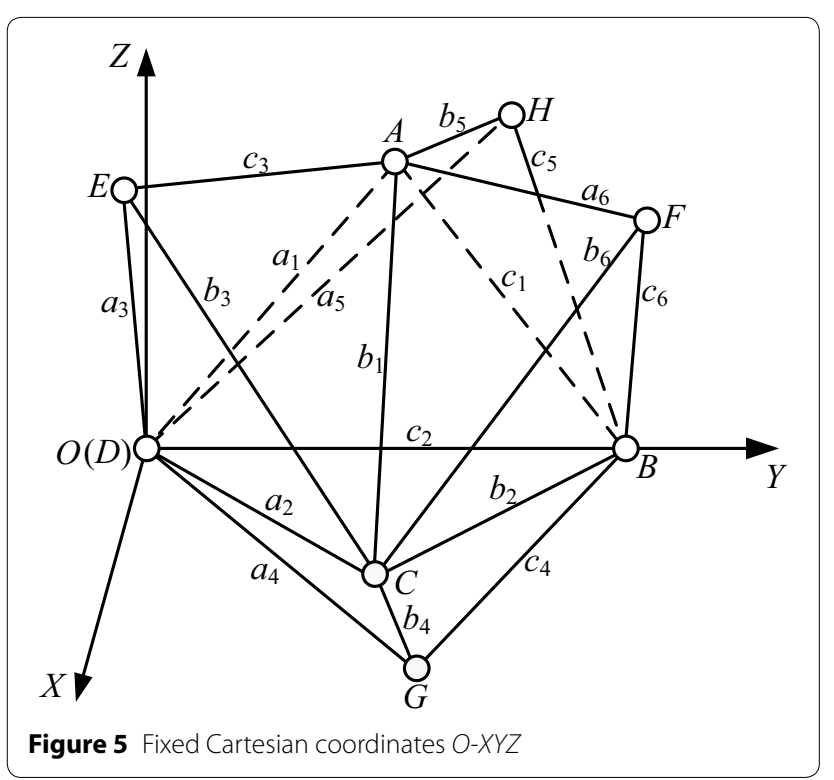


kinematics. For example, the position coordinates of node $A$ is wanted, and the positions of nodes $B, C, D$ and the lengths of sides $A B, A C, A D$ are all known, then the key could be solved by a ternary quadratic group, as Eq. (4) shown. And the results are described in Eq. (5).

$$
\begin{aligned}
& \left\{\begin{array}{l}
\left({ }_{A}^{O} x-{ }_{D}^{O} x\right)^{2}+\left({ }_{A}^{O} y-{ }_{D}^{O} y\right)^{2}+\left({ }_{A}^{O} z-{ }_{D}^{O} z\right)^{2}=a_{1}^{2}, \\
\left({ }_{A}^{O} x-{ }_{C}^{O} x\right)^{2}+\left({ }_{A}^{O} y-{ }_{C}^{O} y\right)^{2}+\left({ }_{A}^{O} z-{ }_{C}^{O} z\right)^{2}=b_{1}^{2}, \\
\left({ }_{A}^{O} x-{ }_{B}^{O} x\right)^{2}+\left({ }_{A}^{O} y-{ }_{B}^{O} y\right)^{2}+\left({ }_{A}^{O} z-{ }_{B} z\right)^{2}=c_{1}^{2},
\end{array}\right. \\
& { }_{\mathrm{A}}^{O} \mathbf{P}=\left(\begin{array}{c}
O \\
{ }_{A}^{O} x \\
{ }_{A}^{O} y \\
{ }_{A} z
\end{array}\right)=\left(\begin{array}{c}
\frac{{ }_{C}^{O} x^{2}+{ }_{C}^{O} y^{2}+a_{1}^{2}-b_{1}^{2}-{ }_{C}^{O} y_{A}^{O} y}{2} \\
\frac{a_{1}^{2}+c_{2}^{2}-c_{1}^{2}}{2 c_{2}} \\
\sqrt{a_{1}^{2}-{ }_{A}^{O} x^{2}-{ }_{A}^{O} y^{2}}
\end{array}\right) .
\end{aligned}
$$

Similarly with node $A$, node $H$ has special geometric relationships with nodes $A, B$ and $D$, which make the position coordinates of node $H$ be solved by another ternary quadratic group, as Eq. (6) shown.

$$
\left\{\begin{array}{l}
\left({ }_{H}^{O} x-{ }_{D}^{O} x\right)^{2}+\left({ }_{H}^{O} y-{ }_{D}^{O} y\right)^{2}+\left({ }_{H}^{O} z-{ }_{D}^{O} z\right)^{2}=a_{5}^{2}, \\
\left({ }_{H}^{O} x-{ }_{B}^{O} x\right)^{2}+\left({ }_{H}^{O} y-{ }_{B}^{O} y\right)^{2}+\left({ }_{H}^{O} z-{ }_{B}^{O} z\right)^{2}=c_{5}^{2}, \\
\left.{ }_{H}^{O} x-{ }_{A}^{O} x\right)^{2}+\left({ }_{H}^{O} y-{ }_{A}^{O} y\right)^{2}+\left({ }_{H}^{O} z-{ }_{A}^{O} z\right)^{2}=b_{5}^{2} .
\end{array}\right.
$$

The position coordinates of node $H$ are obtained as shown in Eq. (7):

$$
\mathbf{H}_{\mathbf{H}} \mathbf{P}=\left(\begin{array}{c}
O \\
H^{O} \\
O \\
H \\
O \\
H^{H} z
\end{array}\right)=\left(\begin{array}{c}
-\frac{{ }_{A}^{O} z \cdot{ }_{H}^{O} z}{O^{O}}-\frac{R_{1}}{2} \\
2_{A}^{O} x \\
\frac{a_{5}^{2}+c_{2}^{2}-c_{5}^{2}}{2 c_{2}} \\
-\frac{{ }_{A}^{O} z R_{1}}{2_{A}^{O} x^{2} R_{2}}+\frac{R_{3}}{2 R_{2}}
\end{array}\right) .
$$

Where $R_{1}=b_{5}^{2}-a_{5}^{2}-{ }_{A}^{O} x^{2}-{ }_{A}^{O} y^{2}-{ }_{A}^{O} z^{2}+2{ }_{A}^{O} y \cdot{ }_{H}^{O} y$, $R_{2}=\frac{{ }_{A}^{O} z^{2}}{{ }_{A} x^{2}}+1$

$R_{3}=\sqrt{\frac{R_{1}^{2 O} z^{2}}{{ }_{A}^{O} x^{4}}-4 R_{2}\left(\frac{R_{1}^{2}}{4_{A}^{O} x^{2}}+{ }_{H}^{O} y^{2}-a_{5}^{2}\right)}$.

The same goes for the rest nodes $E, F$ and $G$, which could also obtain its position coordinates from the corresponding ternary quadratic groups according to nodes $A$, $C$, $D$, nodes $A, B, C$, and nodes $B, C, D$, respectively.

\subsection{Dynamics of the Tipping Motion}

The tipping motion of 5-TET can be divided into three phases as before tipping, tipping, and contact with the ground. Taking tipping over axis $E G$ by extending struts $\mathrm{AD}$ and $\mathrm{BD}$ as example, the tipping process is shown in Figure 6.

The first phase is to manipulate the controlled nodes to generate the tipping motion. The actuating forces from $\boldsymbol{D A}$ and $\boldsymbol{D B}$ are exerted on nodes $A$ and $B$ during this phase while the base nodes are placed on the ground. The second phase comprises a tipping motion when the base node $D$ leaves the ground and the new node $C$ falls down to the ground. And 5-TET turns into the third phase after node $C$ hits the ground. Assume that all the actuators are locked in this process, in other words, 5-TET can be regarded as a rigid body during the tipping motion.

\subsubsection{Before Tipping Phase}

Dynamically, the critical condition of the tipping is that the ground support force equals to zero, which corresponds to that the $\mathrm{CM}$ goes on the tipping axis in kinematics.

Before tipping motion, the ground support force keeps in positive. The free body diagram in this phase is shown in Figure 7. By applying Euler's moment equation, shown in Eq. (8), with respect to the tipping axis of $E G$, the relation of the nodes are obtained as Eq. (9).

$$
\sum M_{x}=I \alpha,
$$

$$
\begin{aligned}
& \left(m_{A} d_{A}^{y}+m_{B} d_{B}^{y}-m_{C} d_{C}^{y}+m_{D} d_{D}+m_{F} d_{F}^{y}+m_{H} d_{H}^{y}\right) g \\
& \quad-R_{D}^{z} d_{D}=m_{A}\left(\ddot{P}_{A}^{z} d_{A}^{y}-\ddot{P}_{A}^{y} d_{A}^{z}\right)+m_{B}\left(\ddot{P}_{B}^{z} d_{B}^{y}-\ddot{P}_{B}^{y} d_{B}^{z}\right) \\
& \quad+m_{C}\left(\ddot{P}_{C}^{y} d_{C}^{z}-\ddot{P}_{C}^{z} d_{C}^{y}\right)+m_{D} \ddot{P}_{D}^{z} d_{D} \\
& \quad+m_{F}\left(\ddot{P}_{F}^{y} d_{F}^{z}+\ddot{P}_{F}^{z} d_{F}^{y}\right)+m_{H}\left(\ddot{P}_{H}^{z} d_{H}^{y}-\ddot{P}_{H}^{y} d_{H}^{z}\right),
\end{aligned}
$$

where $R_{D}^{z}$ is the $z$ component of $R_{D}$. And the tipping motion starts when $R_{D}^{z}$ becomes negative.

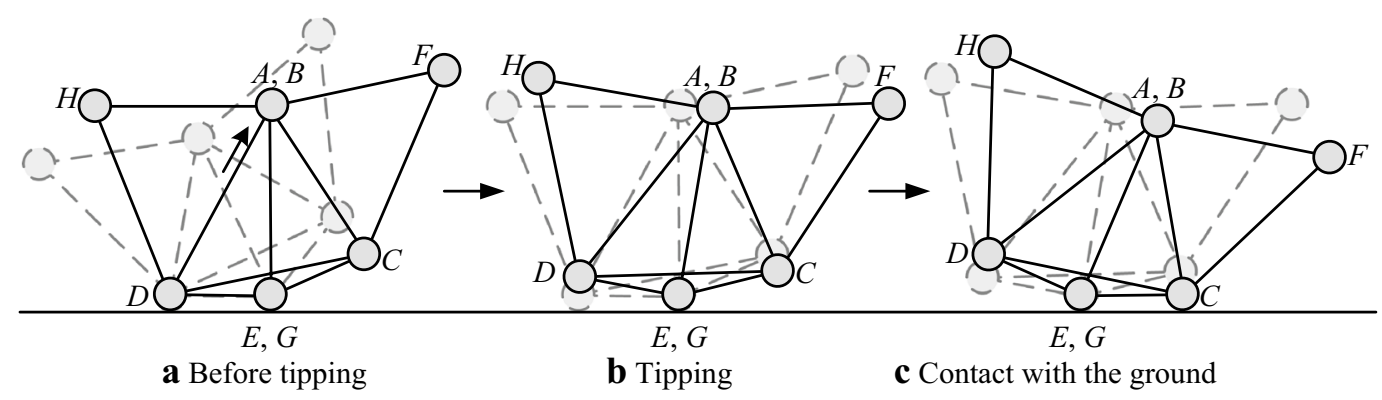

Figure 6 Three phases of tipping motion 


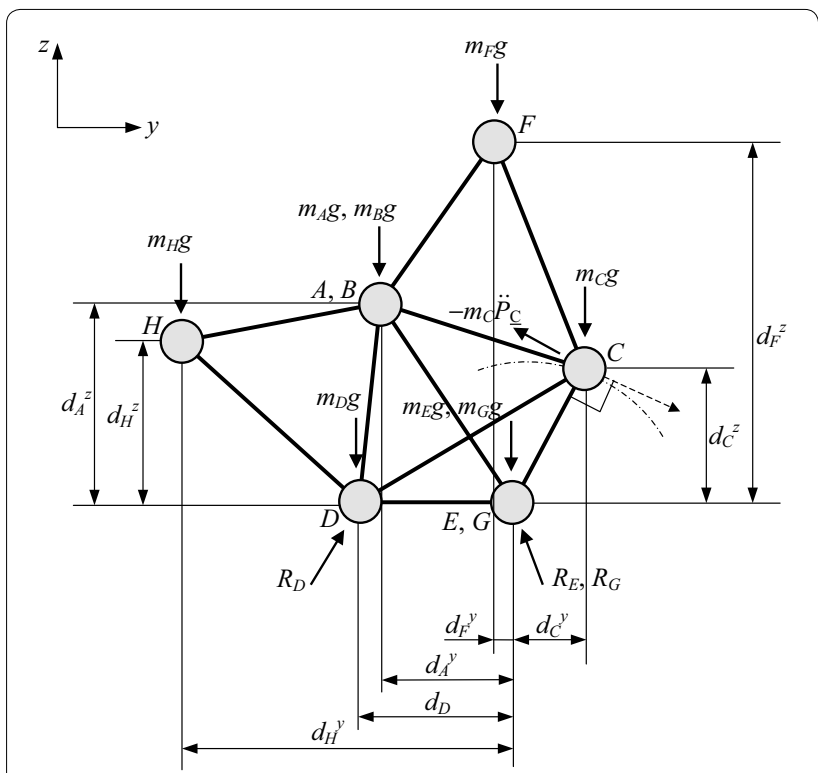

Figure 7 Free body diagram of before tipping phase

The critical tipping condition of 5-tetrahedon is shown in Eq. (10), which reveals that the tipping motion relies on the displacement as well as the acceleration of the nodes.

$R_{D}^{z}=\frac{1}{d_{D}}\left(\begin{array}{l}m_{A} g d_{A}^{y}+m_{B} g d_{B}^{y}-m_{C} g d_{C}^{y} \\ +m_{D} g d_{D}+m_{F} g d_{F}^{y}+m_{H} g d_{H}^{y} \\ -m_{A}\left(\ddot{P}_{A}^{z} d_{A}^{y}-\ddot{P}_{A}^{y} d_{A}^{z}\right)-m_{B}\left(\ddot{P}_{B}^{z} d_{B}^{y}-\ddot{P}_{B}^{y} d_{B}^{z}\right) \\ -m_{C}\left(\ddot{P}_{C}^{y} d_{C}^{z}-\ddot{P}_{C}^{z} d_{C}^{y}\right)-m_{D} \ddot{P}_{D}^{z} d_{D} \\ -m_{F}\left(\ddot{P}_{F}^{y} d_{F}^{z}+\ddot{P}_{F}^{z} d_{F}^{y}\right)-m_{H}\left(\ddot{P}_{H}^{z} d_{H}^{y}-\ddot{P}_{H}^{y} d_{H}^{z}\right)\end{array}\right)<0$.

Figure 8 shows the values of $R_{D}^{z}$ and $P_{\mathrm{CM} x}$ in different accelerations of actuators, which correspond to the tipping conditions in dynamics and kinematics, respectively. The figure expresses that the CM exceeds the tipping axis $E G$ before the support force $R_{D}^{z}$ becomes to zero. And the reason of this result is that the actuating forces from $D A$ and $\boldsymbol{D B}$ not only react on nodes $A$ and $B$, but also on $D$.

\subsubsection{Tipping Phase}

For 5-TET can be regarded as a rigid body during the tipping motion, the system was converted to an inverted pendulum at a pivot joint on the ground, as Figure 9 shown.

The initial angular velocity and the new dynamic parameters can be calculated using the law of conservation of angular momentum. If we assume that the angular momentum is conserved during the instantaneous time of lifting off node $D$, then the angular momentum equations before and after the lifting off can be expressed as follows:

$$
\sum_{i=A}^{H}\left(\mathbf{r}_{i} \times m_{i} \dot{P}_{i}\right)=\mathbf{r}_{\mathrm{CM}} \times m_{\mathrm{CM}} \dot{P}_{\mathrm{CM}},
$$

where $\boldsymbol{r}_{i}$ denotes the position vector of the node from the pivot point $\left(\boldsymbol{P}_{\mathrm{E}}+\boldsymbol{P}_{\mathrm{G}}\right) / 2$. The initial linear velocity $\dot{P}_{\mathrm{CM}}$ can be gotten by rearranging Eq. (11). Then the initial angular velocity of an equivalent system can be obtained:

$$
\dot{\theta}_{\mathrm{CM}}=\frac{\dot{P}_{\mathrm{CM}}}{l_{\mathrm{CM}}} .
$$

The dynamic equation of this system can be expressed as a second order nonlinear equation with initial conditions.

$$
\begin{aligned}
& m_{\mathrm{CM}} l_{\mathrm{CM}}^{2} \ddot{\theta}+m_{\mathrm{CM}} g l_{\mathrm{CM}} \cos \theta=0, \\
& l_{\mathrm{CM}}(0)=l_{\mathrm{CM}_{0}}, \theta(0)=\theta_{\mathrm{CM}_{0}}, \dot{\theta}(0)=\dot{\theta}_{\mathrm{CM}_{0}} .
\end{aligned}
$$

By setting $\dot{\theta}=\frac{\mathrm{d} \theta}{\mathrm{d} t}=s, \ddot{\theta}=\dot{s}=\frac{\mathrm{d} s}{\mathrm{~d} \theta} \mathrm{d} \theta=\frac{\mathrm{d} s}{\mathrm{~d} t} s$, the relation between the angular velocity and rotation angle is obtained by differential equations in Eqs. (15), (16).

$$
\begin{aligned}
& \left\{\begin{array}{l}
\ddot{\theta}=-\frac{g}{l_{\mathrm{CM}}} \cos \theta, \\
\frac{\mathrm{d} s}{\mathrm{~d} \theta}=-\frac{g}{l_{\mathrm{CM}}} \cos \theta, \\
s \mathrm{~d} s=-\frac{g}{l_{\mathrm{CM}}} \cos \theta \mathrm{d} \theta,
\end{array}\right. \\
& \frac{1}{2} s^{2}=\frac{1}{2} \dot{\theta}^{2}=-\frac{g}{l_{\mathrm{CM}}} \sin \theta+\left(\frac{1}{2} \dot{\theta}_{\mathrm{CM}_{0}}^{2}+\frac{g}{l_{\mathrm{CM}_{0}}} \sin \theta_{\mathrm{CM}_{0}}\right) .
\end{aligned}
$$

The angular velocity and acceleration of 5-TET during the tipping phase are shown in Figure 10.

\subsubsection{Contact with the Ground Phase}

In the touchdown phase, the ground is modeled as a spring and damper system as shown in Figure 11. Assuming that the reaction force is $\boldsymbol{R}$, the dynamic equation of the soft contact model is expressed in Eq. (17):

$\mathbf{R}=\mathbf{k} \delta+\mathbf{c} \boldsymbol{B}=\left(\begin{array}{ccc}k_{i}^{x} & 0 & 0 \\ 0 & k_{i}^{y} & 0 \\ 0 & 0 & k_{i}^{z}\end{array}\right)\left(\begin{array}{c}\delta_{i}^{x} \\ \delta_{i}^{y} \\ \delta_{i}^{z}\end{array}\right)+\left(\begin{array}{ccc}c_{x} & 0 & 0 \\ 0 & c_{y} & 0 \\ 0 & 0 & c_{z}\end{array}\right)\left(\begin{array}{c}\dot{\delta}_{i}^{x} \\ \dot{\delta}_{i}^{y} \\ \dot{\delta}_{i}^{z}\end{array}\right)$,

where, $\boldsymbol{k}$ is the spring matrix, $\boldsymbol{c}$ is the damping matrix, and $\boldsymbol{\delta}_{i}$ is the micro displacement in contact moment. Reaction force is proportional to $\boldsymbol{\delta}_{i}$ and $\dot{\delta}_{i}$, and it becomes to zero when the node leaves off the ground.

According to dynamic analysis, 5-TET stays in unstable state in the first two phases, while it is stable in contact with the ground phase. 


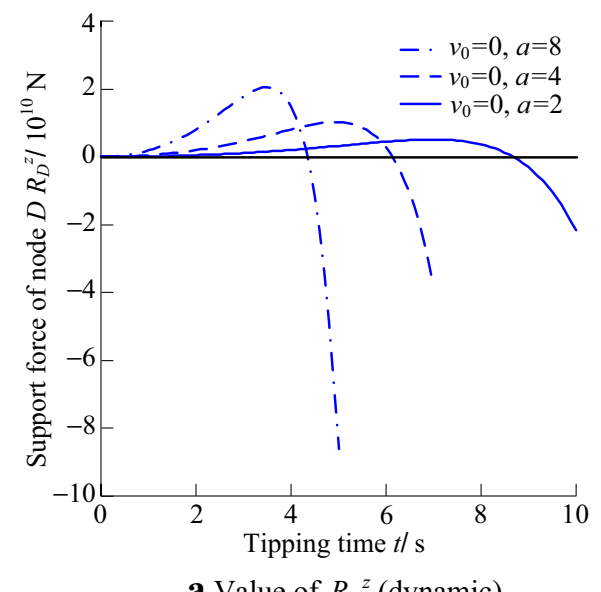

a Value of $R_{D}{ }^{z}$ (dynamic)

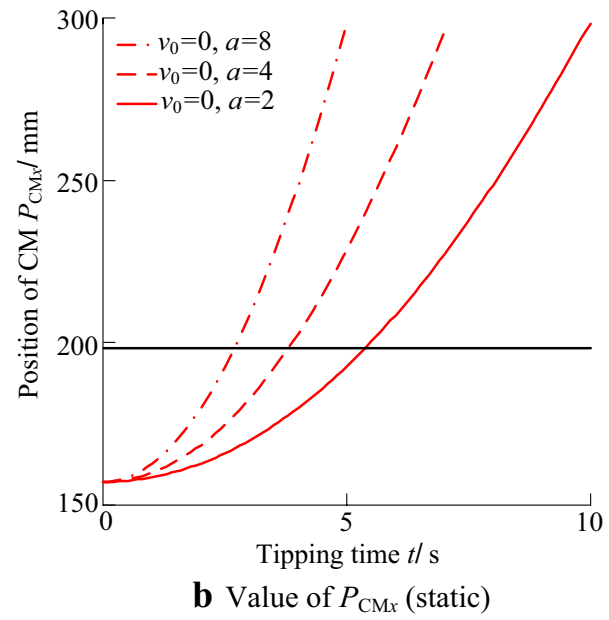

Figure 8 Comparison of dynamic and static tipping conditions

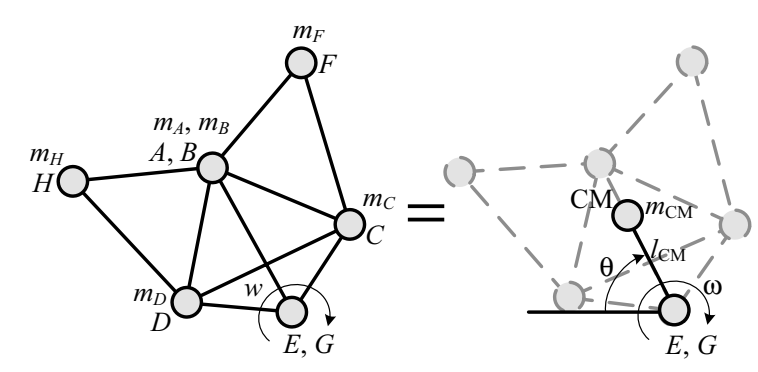

Figure 9 Dynamic model of the tipping phase

\subsection{Motion Gait Optimization for the First Step Rolling}

Based on the isotropic feature of 5-TET, no matter which support area is the initial one, the same result will be gotten. In Figure 3, the initial support area is given as $\triangle \mathrm{DEG}$. The rolling feasibility analysis infers that 5-TET could statically tip towards all of the three directions over edge
$D E, D G$ and $E G$, respectively. Thus, 5-TET is capable of rolling successively by binary codes.

Limited-DOF [31] mechanism is a popular concept in parallel mechanism which has great potential in practical applications with advantages of simple and compact constructions, easy control and low cost. Taking these advantages into the analysis of multi-DOF 5-TET, every step tipping is regarded as the rolling of a limited-DOF mechanism. The objective of the optimization is defined as executing the minimum number of struts. The motion optimization model of 5-TET was set up.

\section{Design vector}

$X=\left[x_{1}, x_{2}, \ldots, x_{18}\right]^{\mathrm{T}}=[A D, A C, A B, D C, B C, B D, E D$, $E C, E A, G D, G C, G B, H D, H A, H B, F A, F C, F B]^{\mathrm{T}}$;

\section{Objective function}

$\min f(\boldsymbol{X})=\sum_{i=1}^{18}\left|x_{i}-x_{i 0}\right|$;

Subject to

$g_{i}(X)=x_{i}\left(x_{i}-1\right)=0,(i=1,2, \ldots, 18)$;

$g_{19}(X)<0$;

$g_{20}(X)<0$.

Where, design variables $x_{i}(i=1,2, \ldots, 18)$ is the present state of actuators that could make 5-TET roll to the specific direction, while $x_{i 0}(i=1,2, \ldots, 18)$ is the former state. The constraint $g_{19}(X)<0$ is the condition that the $\mathrm{CM}$ goes beyond the current support area, while $g_{20}(X)<0$ is the constraint that the CM falls in the new support area.

Note that, during the rolling of 5-TET, the position coordinates of every node change constantly, which increases the difficulty of successive motion analysis. Here, we adopted CM projection on the support planar instead of using coordinates transformation to simplify the kinematic model. Thus, the 19th and 20th constraints were derived by whether the $\mathrm{CM}$ projection and the rest node locate on the same side of the tipping axis. If they were, the cross product between vectors of the two nodes to $\mathrm{CM}$ projection and the cross product between vectors of the two nodes to the rest node would be the same sign, otherwise opposite. The derived process is described as follows:

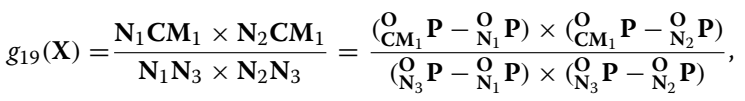

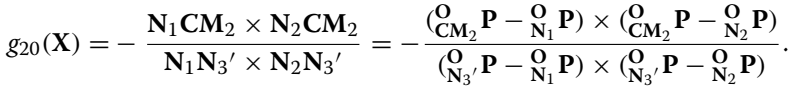

Where $C M_{1}$ is the $C M$ projection on current support area, $C M_{2}$ is the $C M$ projection on the new support area, $N_{1}$ is one node of the tipping axis, $N_{2}$ is the other node of the tipping axis, and $N_{3}$ is the rest node of current support area, while $N_{3}{ }^{\prime}$ is the new node that would touch on the ground. 

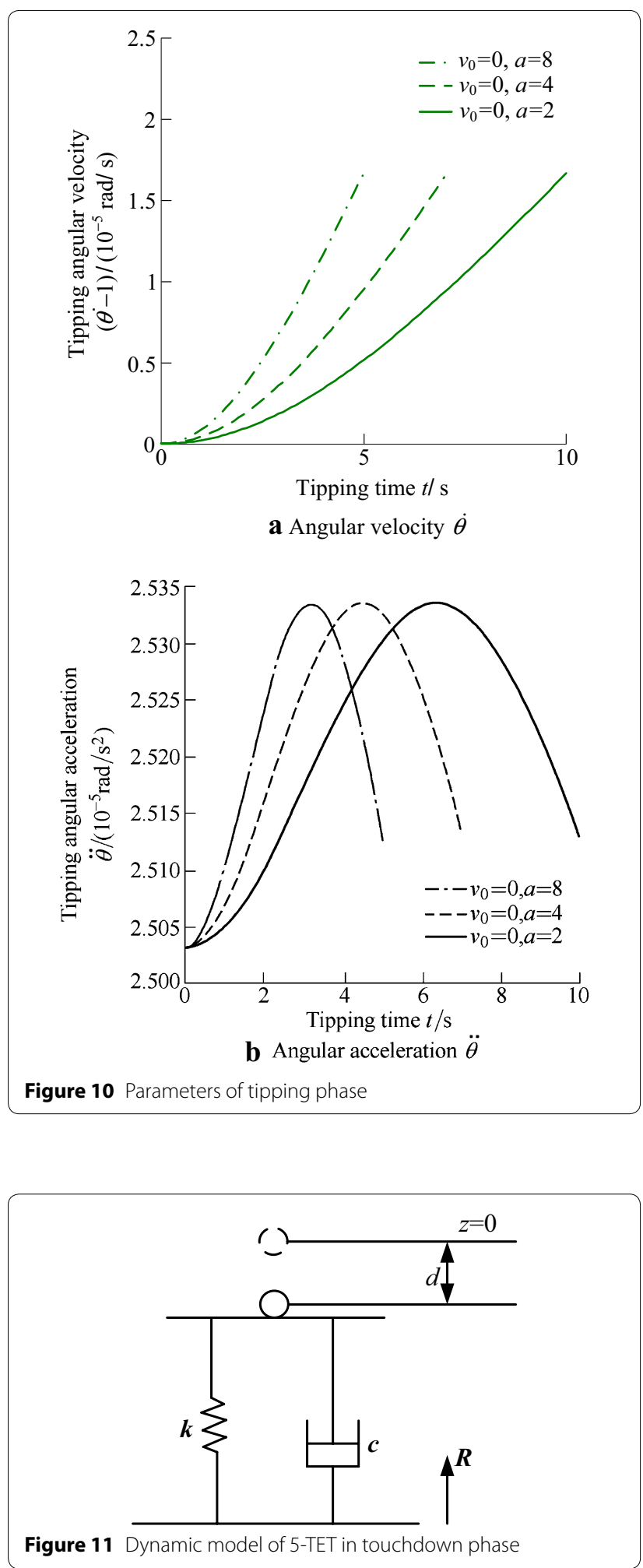

According to the requirement of the optimization model, and considering binary thinking is also applied in GA [15], a GA combined with binary codes is developed to optimize the motion of 5-TET. Where, the crossover and mutation rate are determined by the traditional selection method trial-and-error based on the principle of crossover is expected to be higher while mutation is expected to be lower [32], which respectively equal to 0.2 and 0.02 . According to the genome length of 18 , assumed that the population number is 500 and the maximum generation equals to 50. In GA, the implementation of the objective function and constraints are realized within the fitness function,which is a raw measure of the solution value [33]. For fitness function has no requirement for continuity in the derivatives, virtually any cost function can be selected [14]. Here, to get rapid calculation, the fitness function is defined as Fit $=\mathrm{M}-f(\boldsymbol{X}), \mathrm{M}$ is the maximum possible value of objective function that equals to 18 .

Considering that axis $D E$ and $D G$ are in mirror position, which means these two tipping directions have isotropic feature, so that tipping in Direction I and III are analyzed.

\subsubsection{Tipping in Direction I Over Edge DE}

The tipping step to Direction I over edge $D E$ is optimized, the optimal result is $f\left(\boldsymbol{X}^{*}\right)=3$. There are four different combinations, described as Code 1 to 4 :

Code $1 \quad[0,0,1,0,0,0,0,0,0,0,1,1,0,0,0,0,0,0]$;

Code $2 \quad[0,0,0,0,0,0,0,1,0,0,1,1,0,0,0,0,0,0]$;

Code $3 \quad[0,0,0,0,0,0,0,0,1,0,1,1,0,0,0,0,0,0]$;

Code $4 \quad[0,0,0,0,0,0,0,0,0,0,1,1,0,0,0,0,1,0]$.

The optimal inputs combination of 5-TET is based on the fewest number of executed pneumatic cylinders between two adjacent steps. And fitness function is responsible to find the combinations. The convergence processes of the four optimal solutions are shown in Figure 12. The comparison curves demonstrate that in different inputs combinations, the optimal results are all identical. And the best fitness value is 15 .

Taking the instability before tipping and the stability after tipping into consideration, Code 1 is chosen as the representative of the optimal codes. Figure 13 elaborates the tipping process over edge $D E$ with Code 1 . With the optimal inputs, the projection of CM on the support plane is beyond the support area of $\triangle \mathrm{DEG}$, and the mechanism tips over edge $D E$ until node $H$ touches on the ground, then $\triangle \mathrm{DEH}$ comes to be the new support area.

\subsubsection{Relationship between Direction I and II}

Considering that the initial structure of 5-TET with support area of $\triangle \mathrm{DEG}$, edge $D E$ and $D G$ are in mirror positions, so the pneumatic cylinders that respectively related 


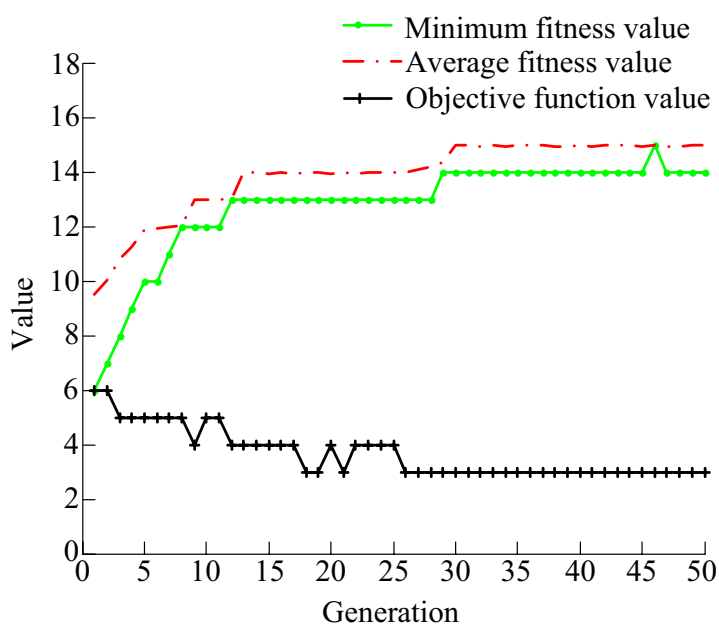

a Code 1

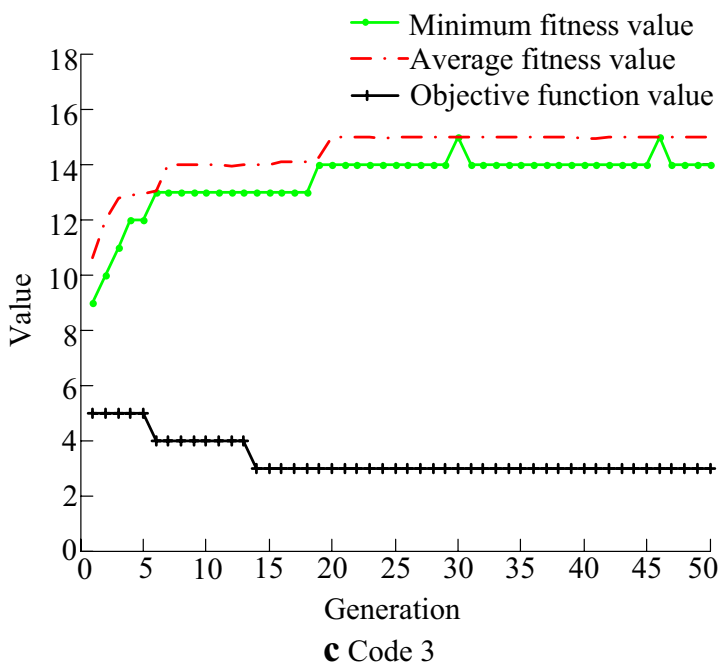

Figure 12 Comparison curves of fitness function (Direction I)
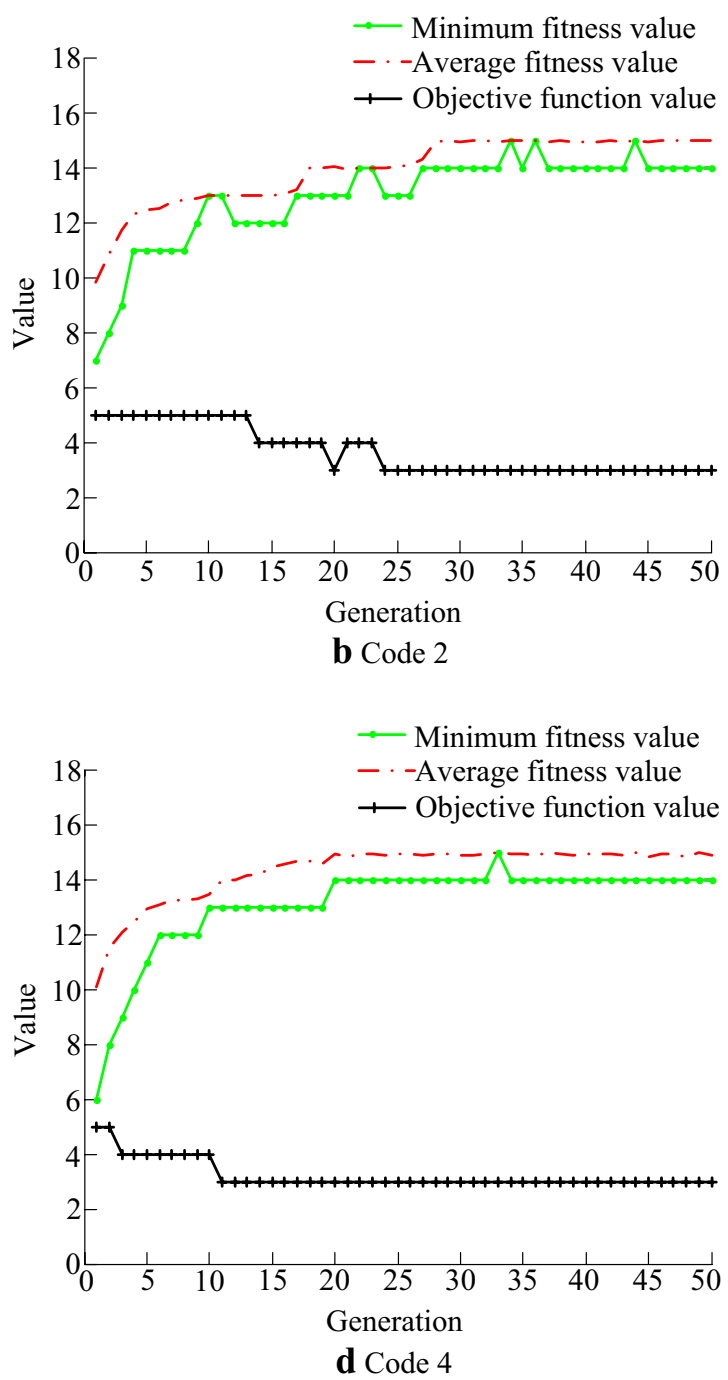

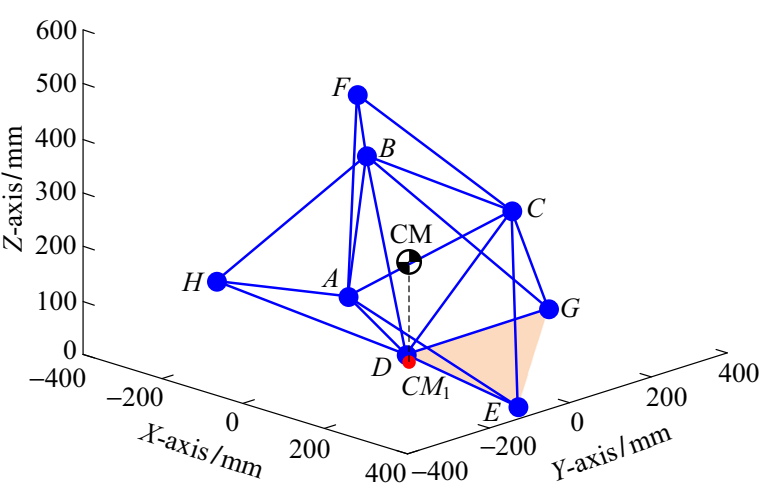

a Code 1: unstable state

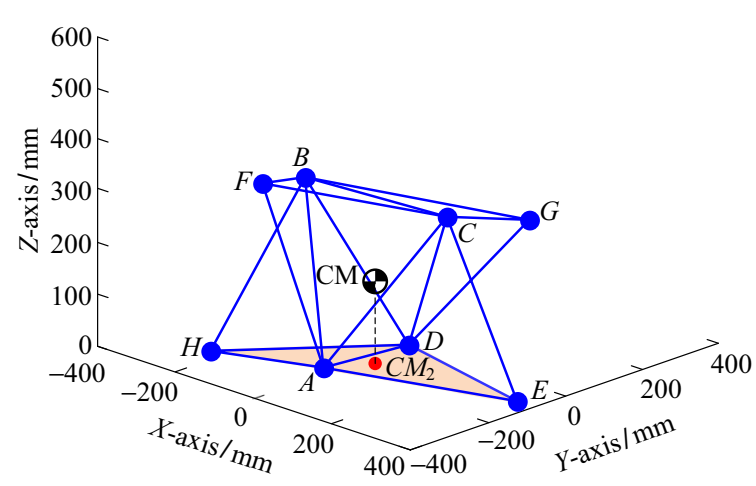

b Code 1: stable state

Figure 13 Tipping process of 5-TET over edge DE with optimal inputs 
to $D E$ and $D G$ are arranged mirrored as well. Here, the correspondence is given in Table 2 .

According to Table 2, four optimal inputs combinations of tipping to Direction II were gotten as Codes 5 to 8 :

Code $5 \quad[0,0,1,0,0,0,0,1,1,0,0,0,0,0,0,0,0,0]$;

Code $6 \quad[0,0,0,0,0,0,0,1,1,0,1,0,0,0,0,0,0,0]$;

Code $7 \quad[0,0,0,0,0,0,0,1,1,0,0,1,0,0,0,0,0,0]$;

Code $8 \quad[0,0,0,0,0,0,0,1,1,0,0,0,0,0,0,0,1,0]$.

Since Code 1 is the representation of tipping in Direction I, Code 5 is chosen to represent the tipping in Direction II. The construction of 5-TET with Code 5 is shown in Figure 14. The CM of the mechanism is beyond the initial support area $\triangle \mathrm{DEG}$, and 5-TET will tip over axis $D G$ until node $H$ hits on the ground, and the next support area is $\triangle \mathrm{DGH}$.

\subsubsection{Tipping in Direction III over Edge EG}

The third tipping direction of 5-TET mechanism is asymmetric with the first two. For the first step rolling by tipping over edge $E G$, the optimal result is $f\left(X^{*}\right)=2$. And there are also four different inputs combinations as follows:

Code $9 \quad[1,0,0,0,0,1,0,0,0,0,0,0,0,0,0,0,0,0]$;

Code $10 \quad[1,0,0,0,0,0,0,0,0,0,1,0,0,0,0,0,0,0]$;

Code $11 \quad[0,0,0,0,0,1,0,1,0,0,0,0,0,0,0,0,0,0]$;

Code $12[0,0,0,0,0,1,0,0,0,0,0,0,0,0,0,1,0,0]$.
The optimal result indicates that 5-TET could tip to Direction III by actuating only two struts. The fitness curves are described in Figure 15, and the fitness functions are all convergent to 16 . Code 10 is taken as the representative code of tipping in Direction III, and the tipping process is described in Figure 16. The projection of $\mathrm{CM}$ on the ground is beyond the support area of $\triangle \mathrm{DEG}$, then the mechanism tips over edge $E G$, and node $C$ is the new support node that would make up the new support area of $\triangle$ CEG.

\subsection{Motion Optimization for Successive Rolling}

It is recognized that multi-DOF mechanisms have the advantage of high mobile flexibility while the complexity of the movement would simultaneously exist. Therefore, the motion simplification and optimization are significant, especially for successive movement. Based on binary control, 5-TET can tip to all the three directions of the initial support area. For isotropic feather, 5-TET can realize successive locomotion with binary control. Assume that the mechanism can not roll back to the last step. Thus, 5-TET has three possible tipping directions only in the first step rolling while it would change to two from the second step. Based on nodes combination principle of triangle support area (one from nodes $A$ to $D$, another two from $E$ to $H$ ) of 5-TET, there are $24\left(C_{4}{ }^{1} \times C_{4}{ }^{2}=24\right)$ possible support triangles obtained by permutation and combination rules. But the real number is only 12 for linkages interference in actual, the possibilities are shown in Table 3.

Table 2 Correspondence of telescopic struts between two mirrored tipping axes

\begin{tabular}{|c|c|c|c|c|c|c|c|c|c|c|c|c|c|c|c|c|c|c|}
\hline Tipping axis & Corr & sponc & ng ed & & & & & & & & & & & & & & & \\
\hline$D E$ & $A D$ & $A C$ & $A B$ & $D C$ & $B C$ & $B D$ & $E D$ & $E C$ & $E A$ & $G D$ & GC & $G B$ & $H D$ & $H A$ & $H B$ & $F A$ & $F C$ & $F B$ \\
\hline$D G$ & $B D$ & $B C$ & $A B$ & $D C$ & $A C$ & $A D$ & $G D$ & GC & $G B$ & $E D$ & $E C$ & $E A$ & $H D$ & $H B$ & $H A$ & $F B$ & $F C$ & $F A$ \\
\hline
\end{tabular}

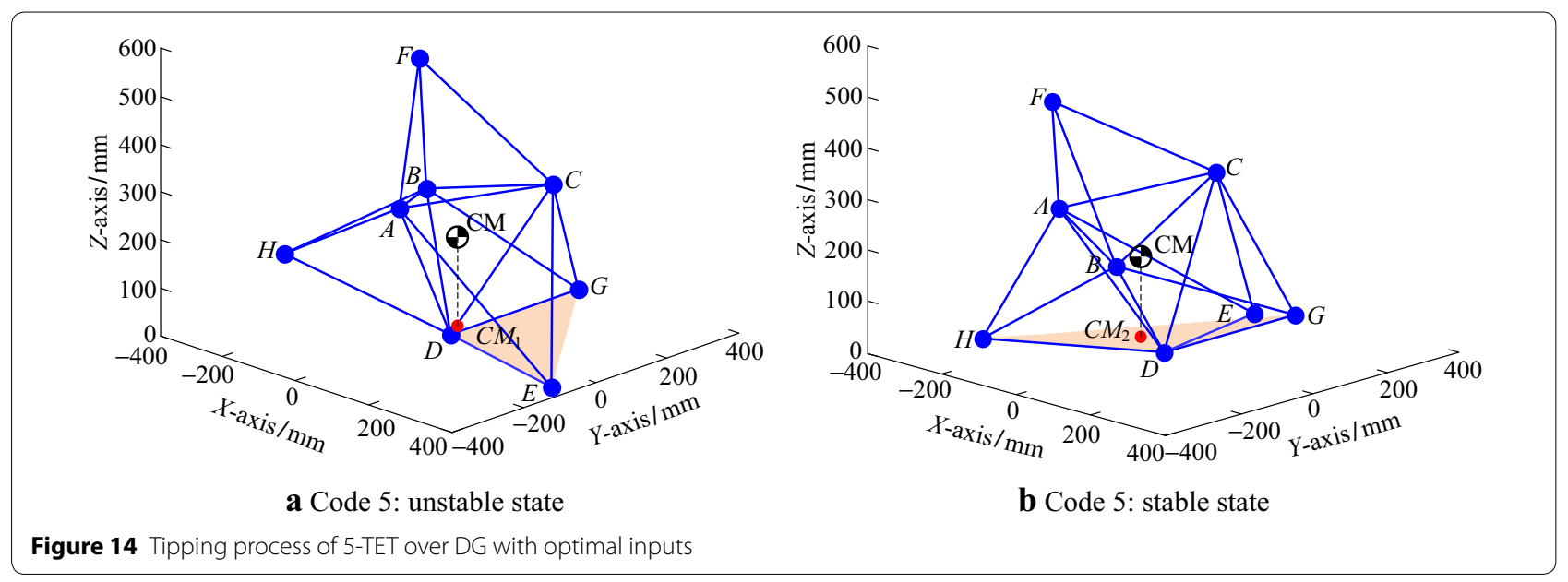




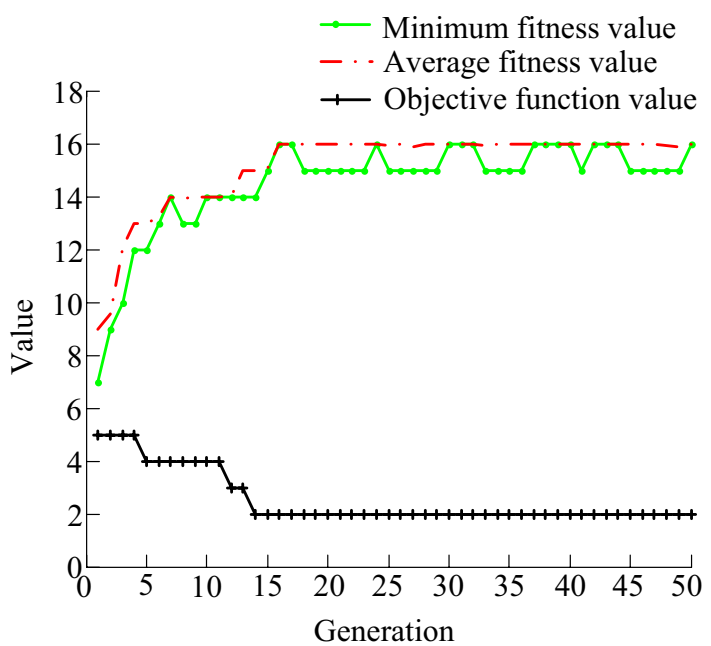

a Code 9

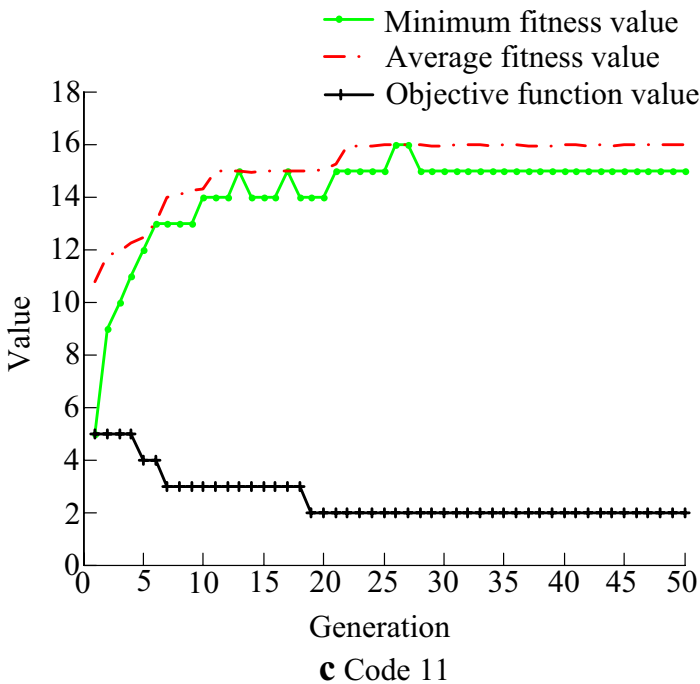

Figure 15 Comparison curves of fitness function (Direction III)
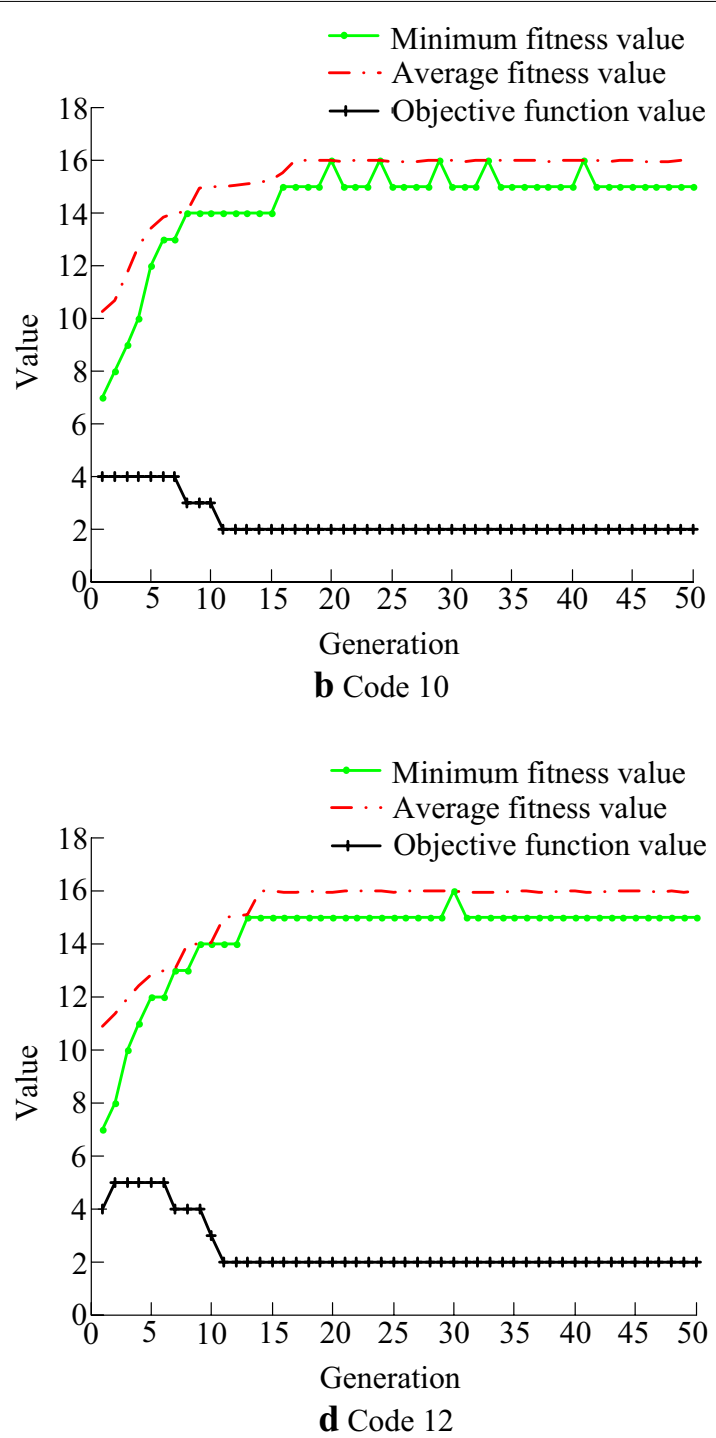

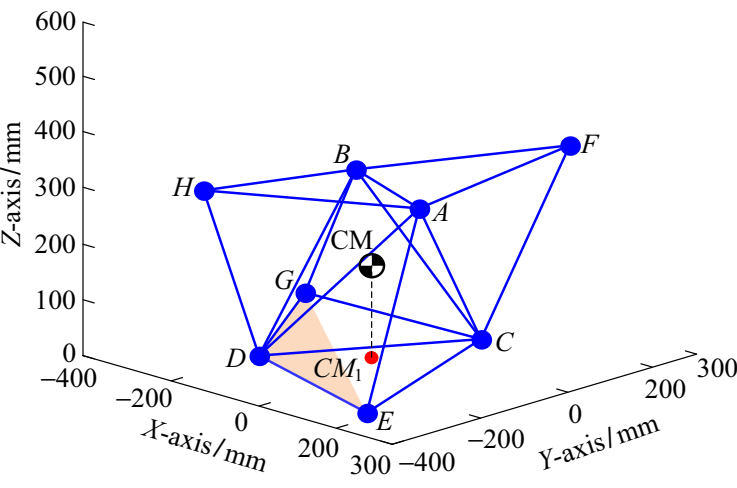

a Code 10: unstable state

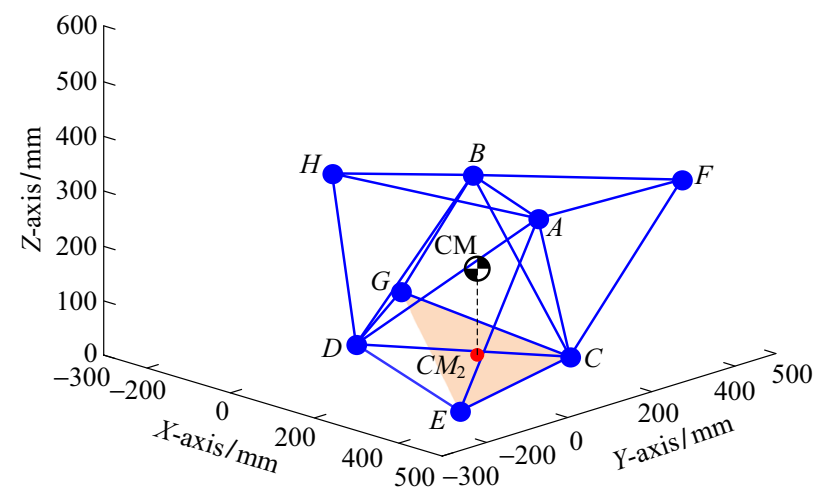

b Code 10: stable state

Figure 16 Tipping process of 5-TET over EG with optimal inputs 
Based on the optimization of single step rolling of 5 -TET, a successive moving optimization algorithm is developed. The objective of the algorithm is to reach the target by executing the least pneumatic cylinders between every two adjacent steps, which requires every step moves with limited-DOF [31]. The tipping axis of each step depends on the intersection of the line segment between target and CM projection on the ground.

5-TET can automatically judge the tipping direction by the tipping rule, and the new touchdown node is also shown automatically according to Table 3 .

For the special rolling mode, the mechanism not only has the motion of straight going, but also turning motion.

\subsubsection{Straight Path}

The targets used to achieve the straight rolling of the algorithm were distributed in four quadrants as follows:

\section{Quadrant I $\quad(1500 \mathrm{~mm}, 2000 \mathrm{~mm})$; \\ Quadrant II $\quad(-1500 \mathrm{~mm}, 2000 \mathrm{~mm})$; \\ Quadrant III $\quad(-1500 \mathrm{~mm},-2000 \mathrm{~mm})$; \\ Quadrant IV $\quad(1500 \mathrm{~mm},-2000 \mathrm{~mm})$.}

The optimal inputs and motion paths are obtained, as Figure 17 shown, where the straight motion paths are expressed by the real support triangles. And the detailed optimal inputs are elaborated in Figure 18. In straight successive motion, the 5-TET rolls to the target with at most 5 executed pneumatic cylinders between every two adjacent steps.

\subsubsection{Turning Path}

According to nodes combination principle of triangle support area, the turning motion can be divided into two cases. Case I turns around nodes $A, B, C$ or $D$; Case II around nodes $E, F, G$ or $H$.

Case I: Based on the initial support area $\triangle \mathrm{DEG}$, the turning center is node $D$. According to the rolling algorithm, the targets were given with the turning radius of $100 \mathrm{~mm}$ :

Target $1 \quad(0 \mathrm{~mm},-100 \mathrm{~mm})$;

Target $2 \quad(-100 \mathrm{~mm}, 0 \mathrm{~mm})$;

Target $3(0 \mathrm{~mm}, 100 \mathrm{~mm})$.

And the program drew the turning paths as Figure 19. There are two modes of Case I turning. The first is a normal path whose turning angle of a cycle is less than 360 degrees. However, the second is a special situation that the mechanism just turns a round, which indicates that the mechanism has position self-reset ability. The detailed optimal inputs of case I turning are elaborated in Figure 20, which reveals that the 5-TET turns in Case I executed at most 5 pneumatic cylinders between each two steps.

Case II: Considering 5-TET has isotropic feature, thus, node $E$ and $G$ are in a group. Here, assume node $G$ as the turning center. According to the demand of the successive motion algorithm, the target points were given with the turning radius of $200 \mathrm{~mm}$, as follows:

Target $1 \quad(200 \mathrm{~mm}, 0 \mathrm{~mm})$;

Target $2(0 \mathrm{~mm}, 200 \mathrm{~mm})$;

Target $3(-200 \mathrm{~mm}, 0 \mathrm{~mm})$;

Target $4(0 \mathrm{~mm},-200 \mathrm{~mm})$.

And the program drew the turning path as Figure 21 shown. The detailed optimal inputs of the turning path are elaborated in Figure 22. Similarly with Case I, 5-TET turns a cycle of Case II by executing at most 5 alternative pneumatic cylinders between each two steps.

From the detailed elaboration of straight paths and turning paths, as Figures 17, 19, and 21 shown, 5-TET can move to the target by executing at most 5 pneumatic cylinders between every two adjacent steps, which indicates that the 5-TET is a 18 DOFs mechanism in total while it is a 5 DOFs mechanism in every step of movement. This feather simplifies the inputs control and program of the rolling, which makes the mechanism have effective foundations for inputs combination strategy.

\section{Optimization-Based Manipulation Planning}

For 5-TET structure, there are four external nodes $E, F, G$ and $H$. And based on the support principle, there are only two of the four external nodes supported on the ground, while the other two TETs are free and unused. For this structural characteristic, we consider to integrate two separate tetrahedrons as a two-finger gripper to manipulate the object. See in Figure 23, the support nodes $D, E$ and $G$ act as the fixed base, the two flying nodes $F$ and $H$ together act as the end-effector of the manipulator.

For gripper manipulators, the gripping range of the end-effector is a major parameter, which has effects on the capacity of gripper manipulation [34]. For 5-TET

Table 3 Possible combinations of triangle support area

\begin{tabular}{|c|c|c|c|c|c|c|c|c|c|c|c|c|}
\hline Support nodes of $A, B, C, D$ & $A$ & $A$ & $A$ & $B$ & $B$ & $B$ & $C$ & $C$ & $C$ & $D$ & $D$ & $D$ \\
\hline upport nodes of $E, F, G, H$ & EF & $E H$ & $\mathrm{FH}$ & $F G$ & $\mathrm{FH}$ & GH & $E F$ & $E G$ & $F G$ & EG & $E H$ & GH \\
\hline Triangle support area & $\triangle \mathrm{AEF}$ & $\triangle \mathrm{AEH}$ & $\triangle \mathrm{AFH}$ & $\triangle \mathrm{BFG}$ & $\triangle \mathrm{BFH}$ & $\triangle \mathrm{BGH}$ & $\triangle C E F$ & $\triangle C E G$ & $\triangle C F G$ & $\triangle \mathrm{DEG}$ & $\triangle \mathrm{DEH}$ & $\triangle \mathrm{DGH}$ \\
\hline
\end{tabular}




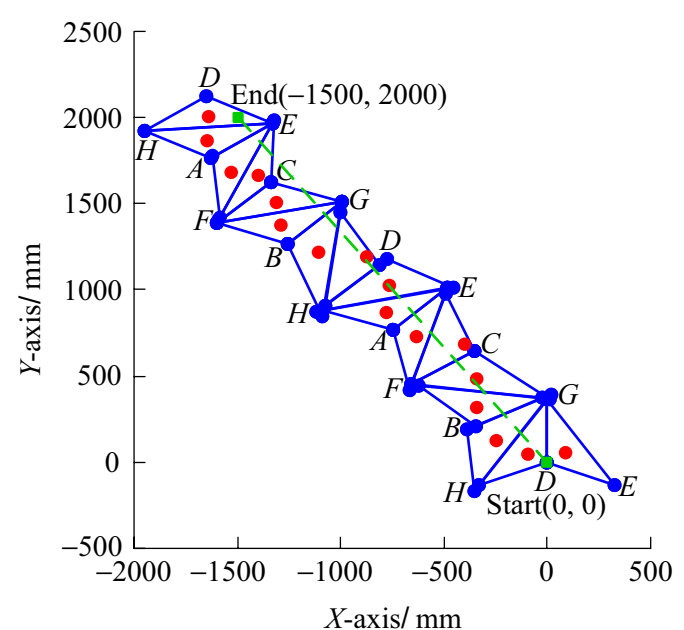

a Quadrant II

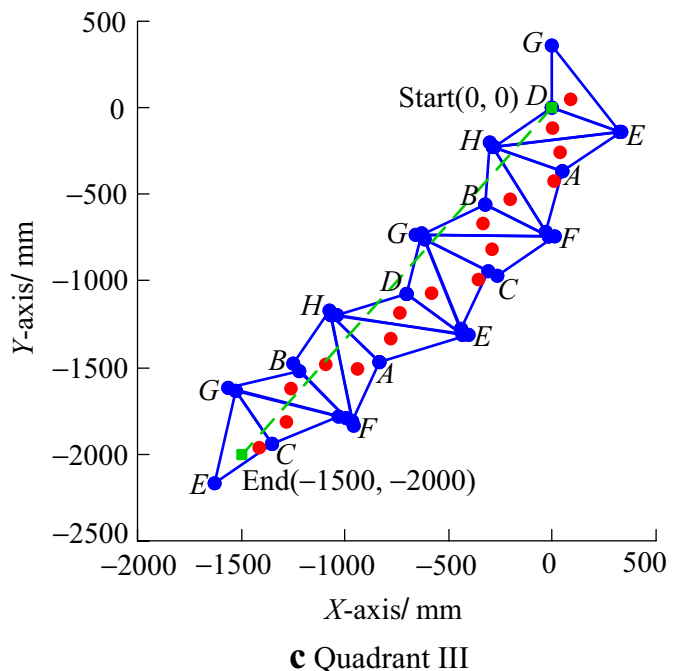

c Quadrant III

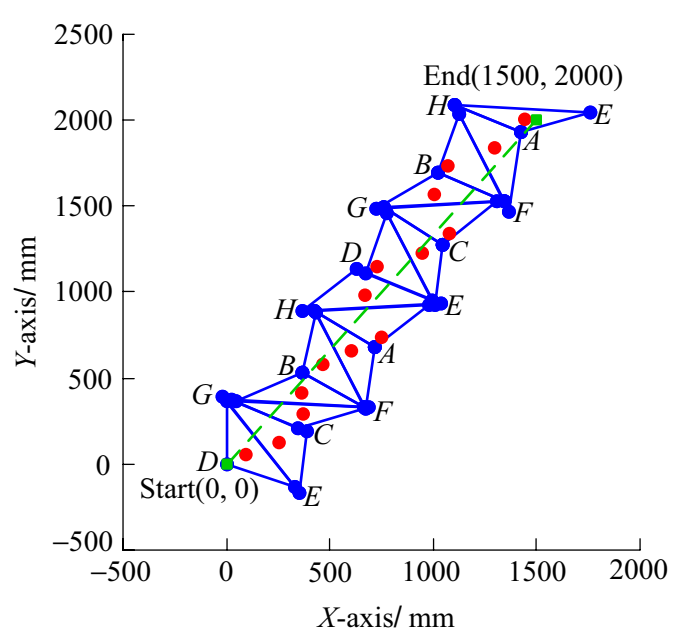

b Quadrant I

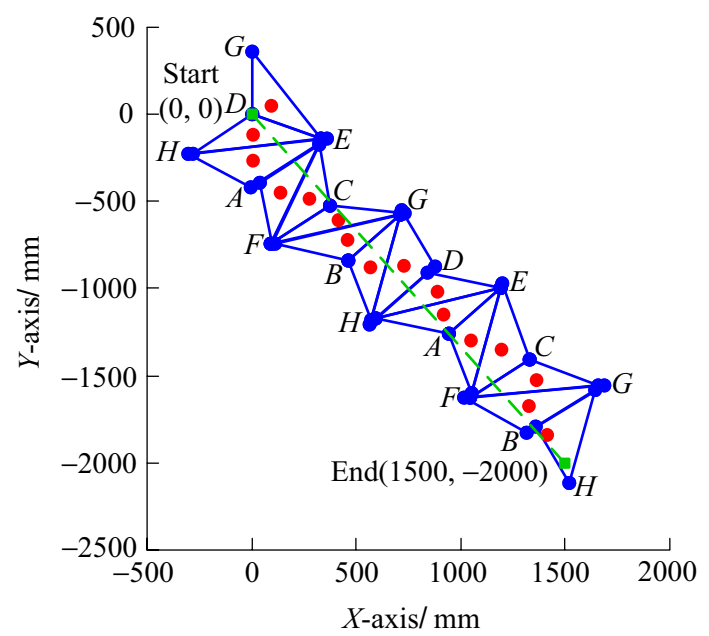

d Quadrant IV

Figure 17 Successive moving paths of 5-TET with optimal inputs

mechanism, the gripping range manifests as the variable range of the dihedral angle of $H-B A-F$. Here, in order to guarantee a stable gripper, nodes $F$ and $H$ should be fully symmetric with strut $\mathrm{AB}$. That makes the variable range of distance between $F$ and $H$ equivalent to the variable range of the dihedral angle $H-B A-F$. In the initial state of 5-TET, the gripper $F$ and $H$ are at a certain distance of $600 \mathrm{~mm}$. However, to realize the gripper action, the dihedral angle $H-B A-F$ should open and close based on the initial state, which would be represented by the distance between nodes $F$ and $H$. The optimization model is set up to get the inputs of the gripper manipulation of 5-TET. Note that, in the optimization model, the number of state change struts is determined as even numbers to maintain symmetry. The optimal model of the gripper is described as follows.

\section{Design vector}

$\boldsymbol{X}=\left[x_{1}, x_{2}, \ldots, x_{18}\right]^{\mathrm{T}}=[A D, A C, A B, D C, B C, B D, E D$, $E C, E A, G D, G C, G B, H D, H A, H B, F A, F C, F B]^{\mathrm{T}}$;

\section{Objective function}

$\min f(\boldsymbol{X})$;

\section{Subject to}

$$
\begin{aligned}
& g_{i}(X)=x_{i}\left(x_{i}-1\right)=0,(i=1,2, \ldots, 18) \text {; } \\
& g_{19}(X)=\sum_{i=1}^{18}\left|x_{i}-x_{i 0}\right|=j,(i=1,2, \ldots, 18 ; j=1,2, \ldots, 18) \text {; }
\end{aligned}
$$

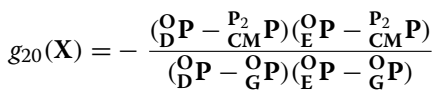

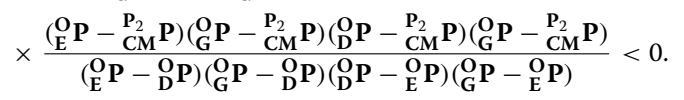

Where $g_{20}(\boldsymbol{X})$ is the constraint of the stability. And similar with the optimal parameters of motion planning, 


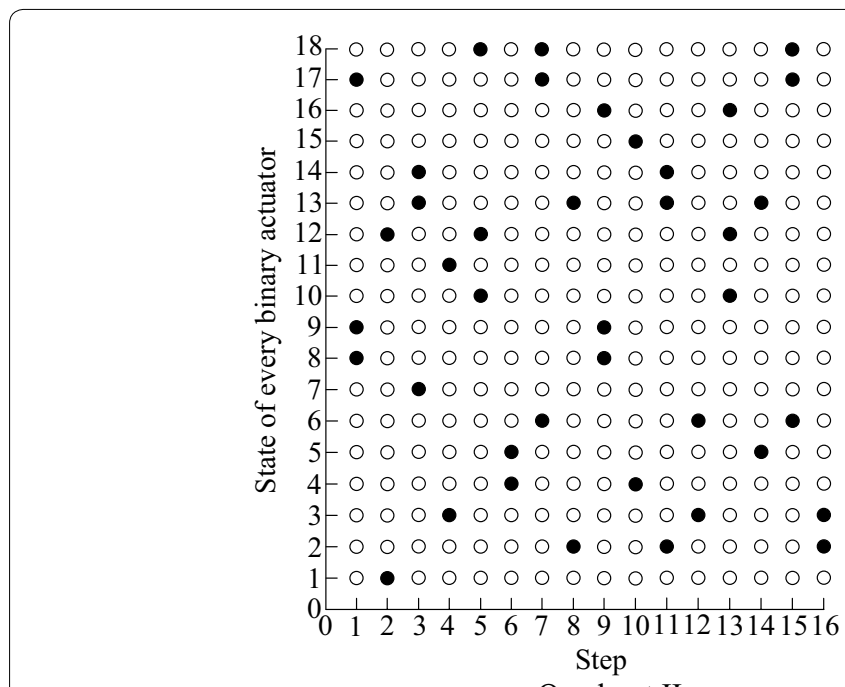

a Quadrant II

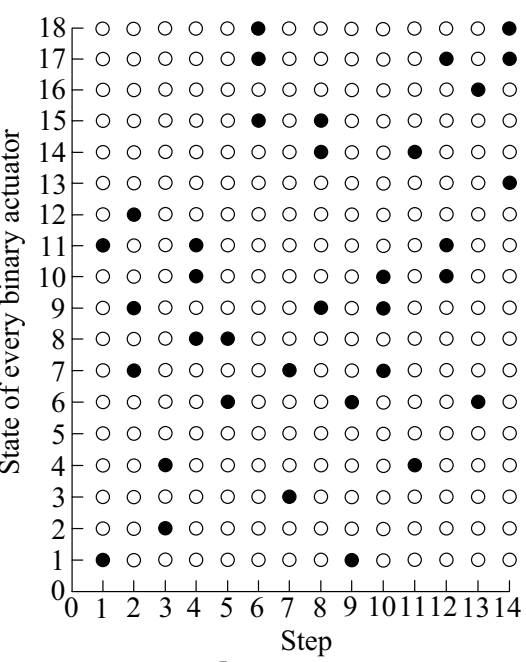

b Quadrant I

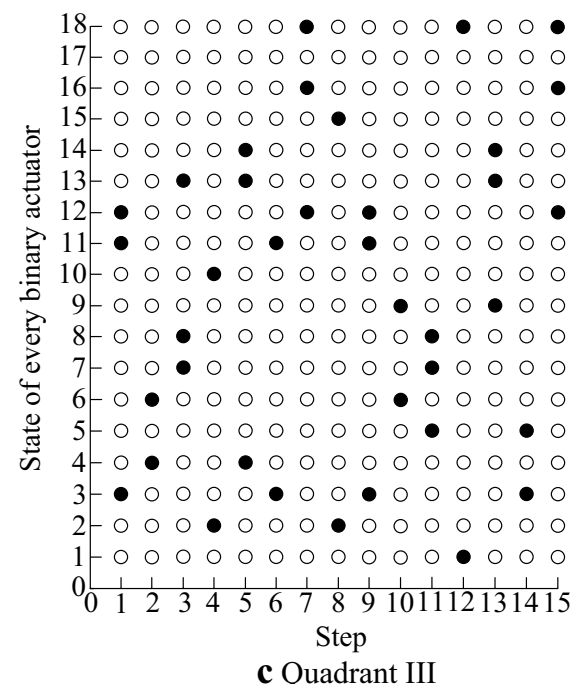

Figure 18 Optimal inputs for successive rolling of 5-TET
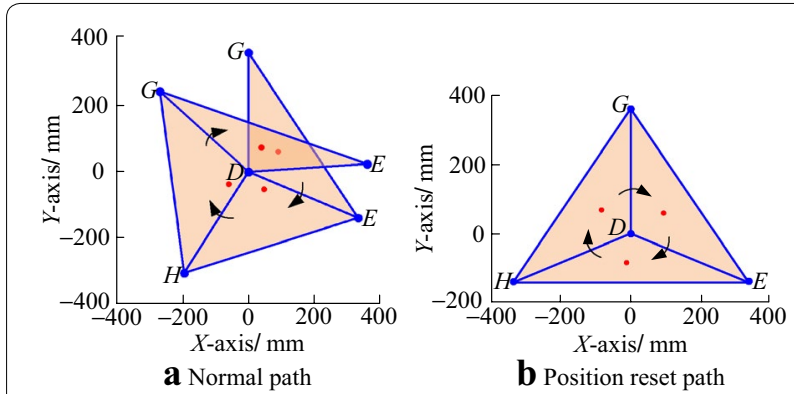

Figure 19 Two turning paths of Case |

the crossover and mutation rates respectively equal to 0.2 and 0.02 , population number is 500 , and the maximum generation is 50. The fitness function is Fit $=M-f(\boldsymbol{X}), M$ is the maximum possible value of the objective function.

\subsection{Manipulation Planning of Opening Wide}

Based on the optimization model of gripper, the opening wide manipulation optimization model is set up. Where $f(\mathbf{X})=-\sqrt{\left({ }_{H}^{O} x-{ }_{F}^{O} x\right)^{2}+\left({ }_{H}^{O} y-{ }_{F}^{O} y\right)^{2}+\left({ }_{H}^{O} z-{ }_{F}^{O} z\right)^{2}}$, and $M=600$. Then the opening wide manipulation was optimized, and the optimal result is $f\left(X^{*}\right)=846.3602$, the number of the lengths altering of pneumatic cylinders is 4, described as Code 13: $[0,0,0,0,0,0,0,0,0,0,0,0$, $0,1,1,1,0,1]$. The fitness function and the stable state mathematical model are depicted as Figure 24. The projection of CM on the ground has been in the support area of $\triangle D E G$ to remain the stability of the overall unit, and the final coordinates of the CM is $[-34.2946,125.7310$, 175.6187]. 


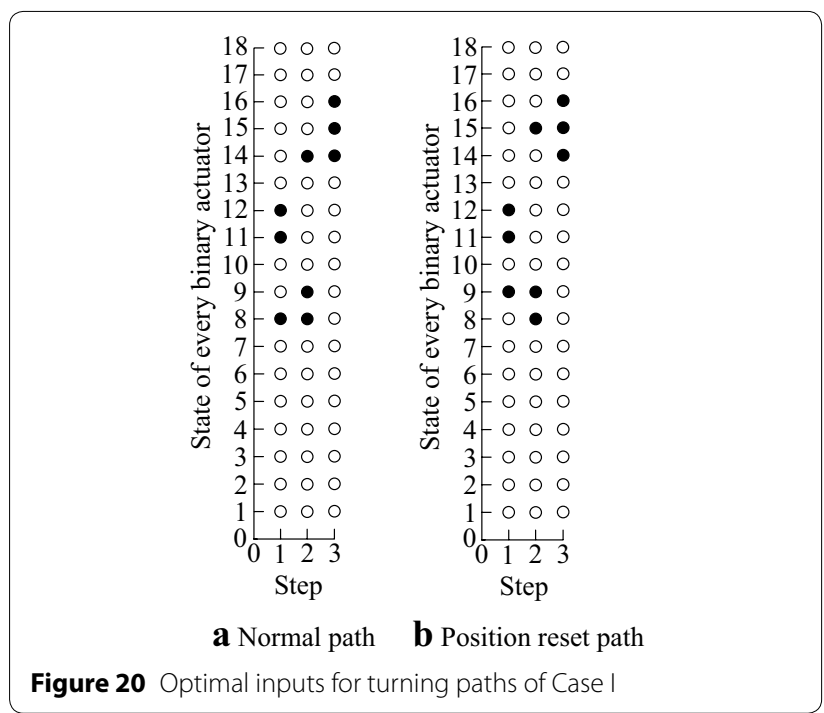

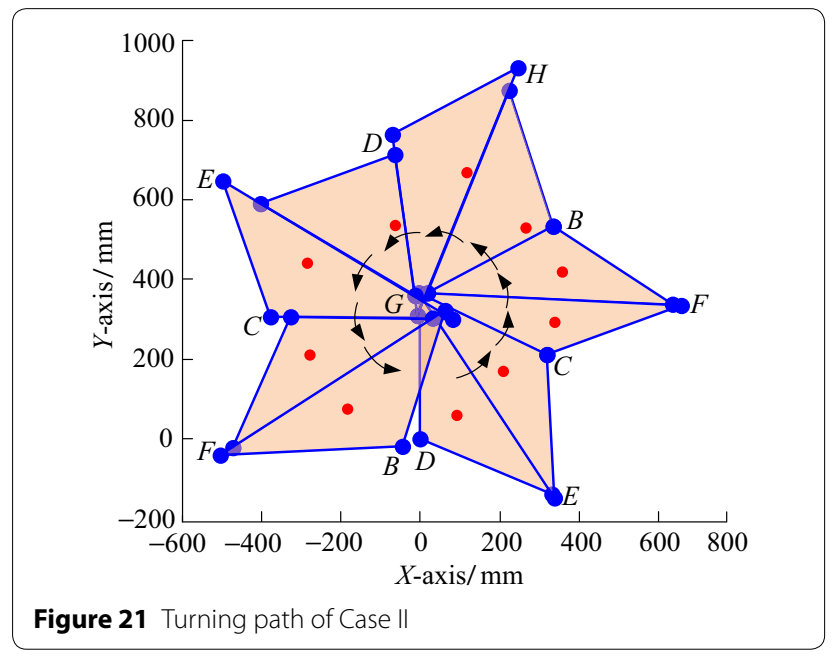

4.2 Manipulation Planning of Turning Down

For the optimization manipulation model of turning down, $f(\mathbf{X})=\sqrt{\left({ }_{H}^{O} x-{ }_{F}^{O} x\right)^{2}+\left({ }_{H}^{O} y-{ }_{F}^{O} y\right)^{2}+\left({ }_{H}^{O} z-{ }_{F}^{O} z\right)^{2}}$. Here, assume that nodes $A, B, F$ and $H$ are coplanar, which means the dihedral angle $H-B A-F$ is at the maximum. And the longest distance between $F$ and $H$ can be easily obtained, $l_{F H \max }=846.64 \mathrm{~mm}$, rounded to $M=$ 850.

The optimal result of turning down manipulation is expressed as $f\left(X^{*}\right)=110.9518$. The number of alternative pneumatic cylinders is four as well. The input code of turning down is obtained as Code $14:[0,0,1,1,0,0,0,0$, $0,0,0,0,1,0,0,0,1,0]$. The fitness function and the stable state mathematical model are shown in Figure 25. The coordinates of the CM is [50.1528, 100.3049, 220.4200]. During the change of the input, the ground projection
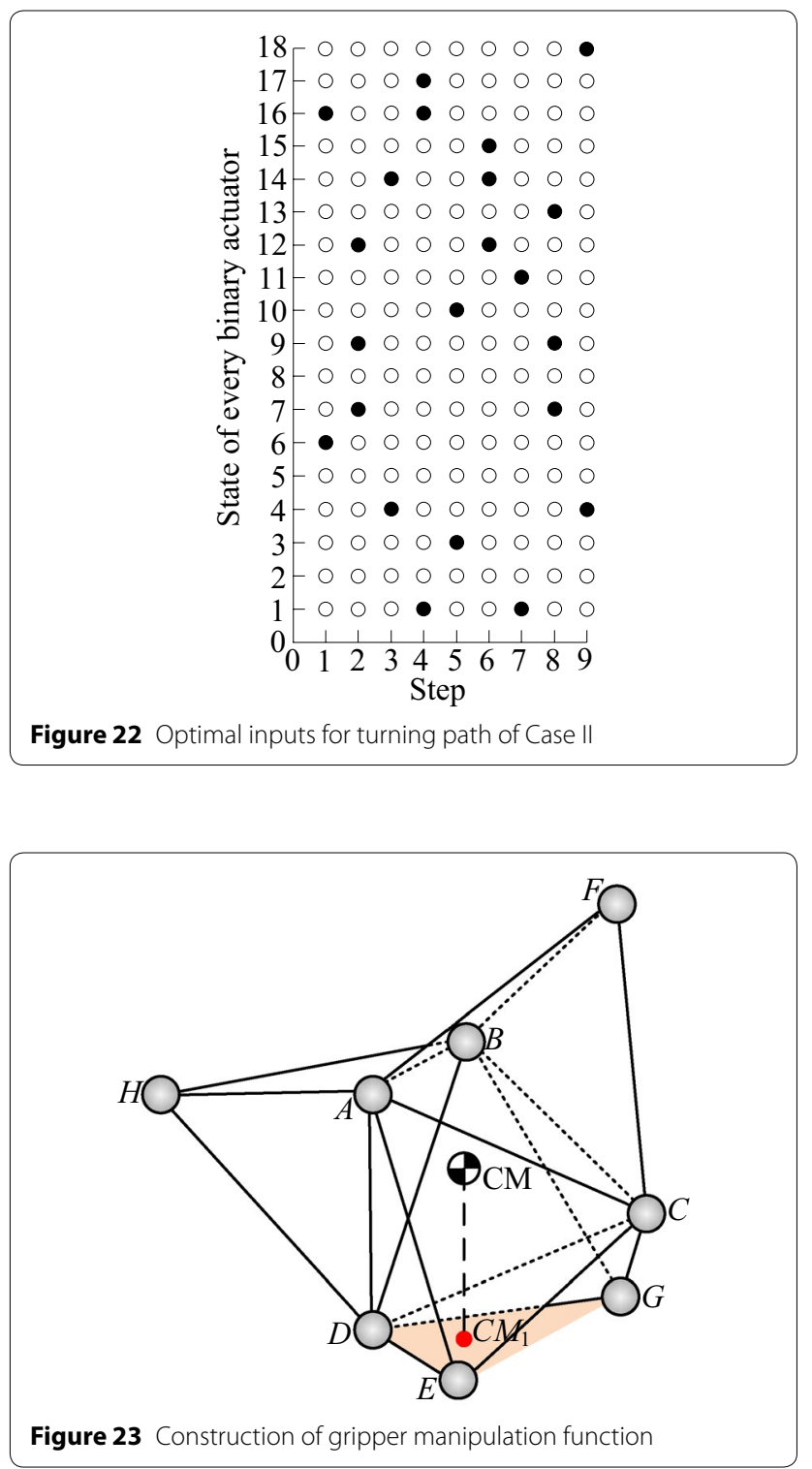

of CM is always in the support area of $\triangle \mathrm{DEG}$, so 5 -TET manipulated in a stable state.

\subsection{Implementation of Gripper Manipulation}

According to the two ultimate gripper states of 5-TET, the intermediate gripper steps could be planned based on binary control strategy as well as the gripper principle that the symmetry of nodes $F$ and $H$. Take $\triangle D E G$ as the initial support area as well, the gripper manipulation was planned, as Figure 26 shown. There are 11 manipulate steps which was made up by 10 different constructions of the binary control 5-TET, the corresponding inputs are detailed in Figure 26(b). And, $\triangle D E G$ is the support triangle of all the 11 steps. But, in the first eight steps, the support nodes $D, E$ and $G$ were fixed, while in the ninth step, 

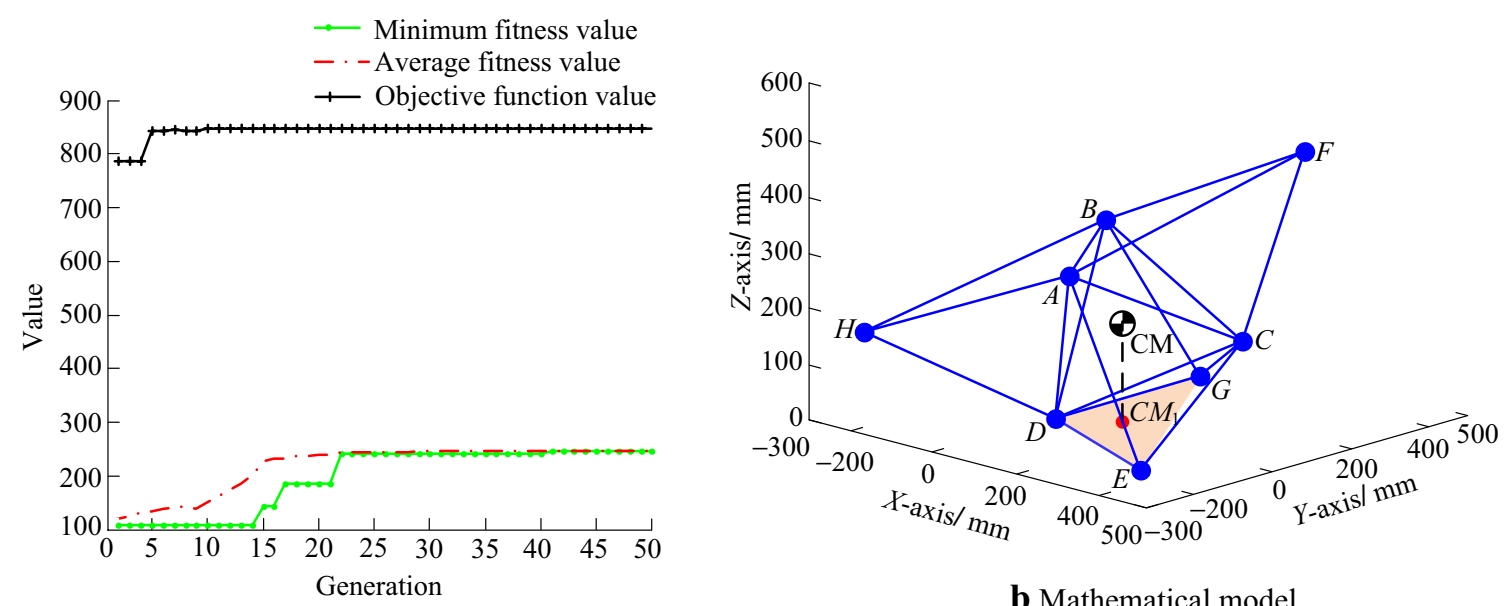

a Fitness function curves

b Mathematical model

Figure 24 Optimal input for opening wide manipulation

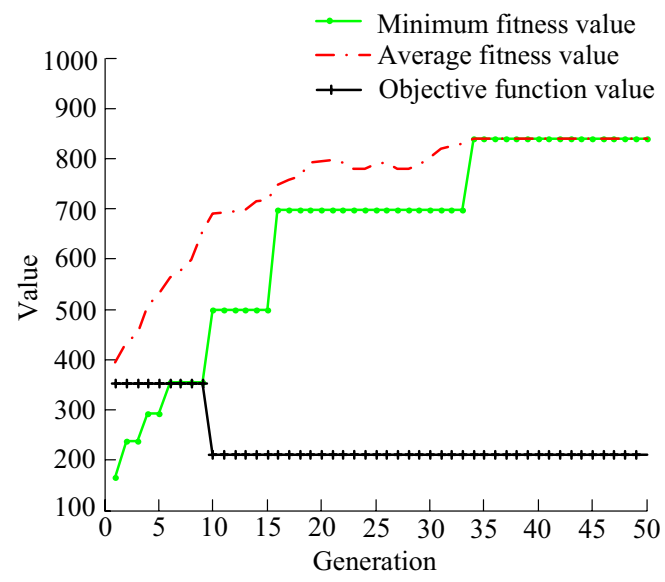

a Fitness function curves

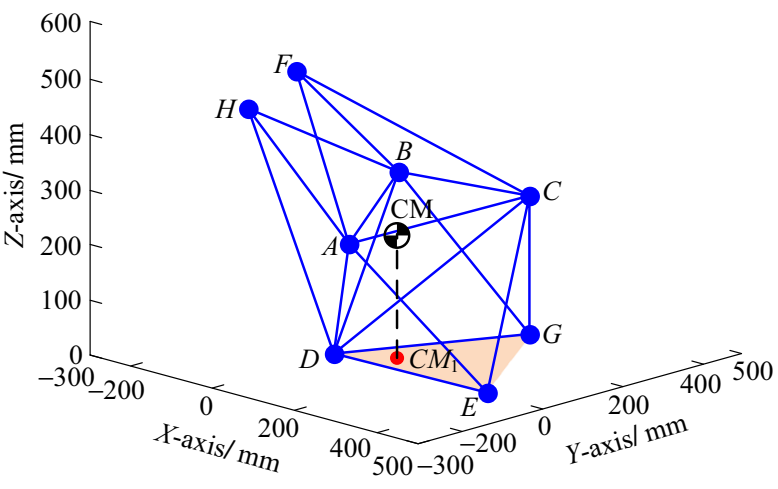

b Mathematical model

Figure 25 Optimal input for turning down manipulation

nodes $E$ and $G$ moved closer to each other, and in the last step, nodes $E$ and $G$ moved even closer (in Figure 26(c)). However, in the whole process of gripper manipulation, the CM projection of 5-TET on the ground was always in the support area, and the majority of $\mathrm{CM}$ projections were in the mid position of the support area, which maintain it stable during gripper manipulations. The planned manipulation workspace is described in Figure 26(d).

In gripper manipulation mode, the number of the executed pneumatic cylinders between every two adjacent steps is no more than 4 , so that 5-TET mechanism is an 18 DOFs mechanism in total while it is a 4 DOFs mechanism in every step manipulation of gripper. And taking rolling motion into consideration, 5-TET is a 5 DOFs mechanism with rolling locomotion mode and gripper manipulation mode.

\section{Energy Consumption Analysis}

The 5-TET realizes locomotion and manipulation functions by deformation, and the $\mathrm{CM}$ of the mechanism frequently changed by driving actuators, so that the energy and locomotion efficiency may be lower than that of traditional mobile mechanisms, such as wheeled and tracked robots.

Taking wheeled robot as example to be compared with 5 -TET on energy consumption and locomotion efficiency. The total weight of the robot is set to be the same with 5 -TET as $12 \mathrm{~kg}$. And the moving path is set as the same trajectory of going straight from the origin $(0,0)$ to the target $(1500,2000)$ in Quadrant I corresponding to Figure 17(b), a total of $2.5 \mathrm{~m}$. 

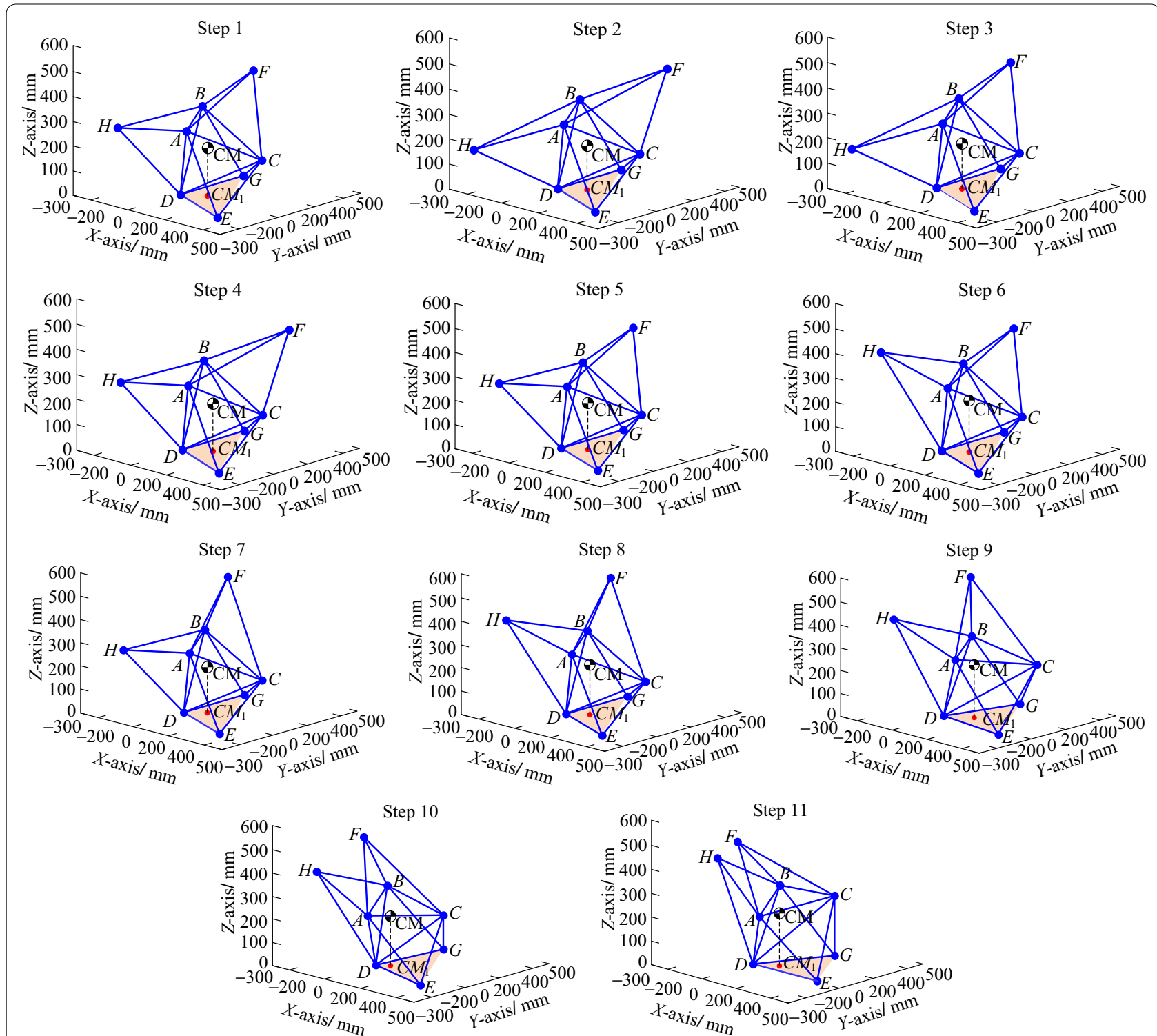

a Gripper manipulation process

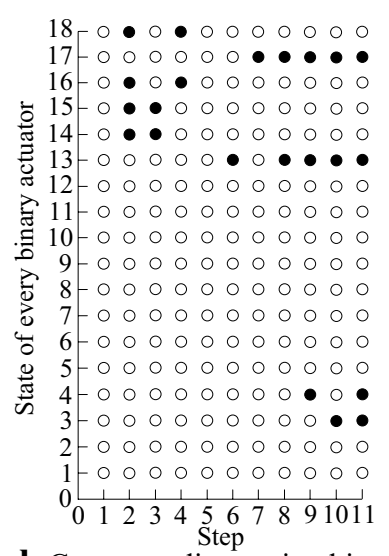

b Corresponding optimal inputs

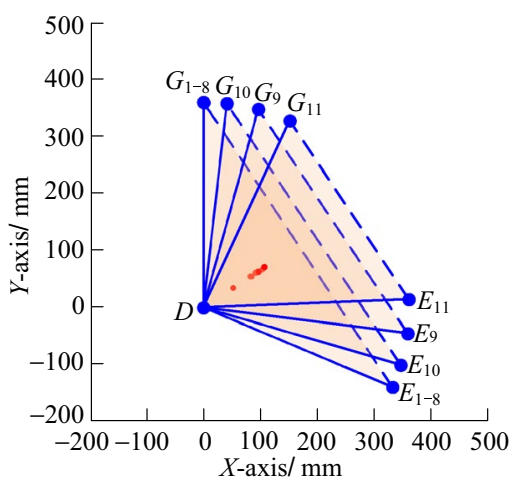

c $\mathrm{CM}$ projections on the ground

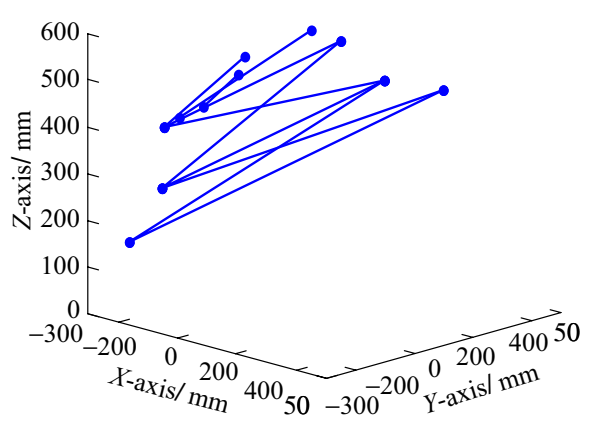

d Workspace

Figure 26 Motion planning of gripper manipulation 


\subsection{The Wheeled Robot}

For the wheeled robot, when it goes forward, it has four forces as gravity $\boldsymbol{G}$, traction force $\boldsymbol{F}_{\text {traction, }}$ ground friction $\boldsymbol{F}_{\text {friction }}$ and air resistance $\boldsymbol{F}_{\text {air }}$, the force diagram is shown in Figure 27.

Assume that this wheeled robot moves at a constant speed, then the acceleration of the system equals to 0 . According to Newton's Second Law, the resultant force $\boldsymbol{F}$ equals to 0, as Eq. (19) shown. Here the air resistance is negligible, so that $\boldsymbol{F}_{\text {traction }}=-\boldsymbol{F}_{\text {friction. }}$. And $\boldsymbol{F}_{\text {friction }}=f \boldsymbol{g}=$ $f m g=0.6 \times 12 \times 9.8=70.56 \mathrm{~N}$. Here, $f$ represents the friction coefficient between automobile tires and dry asphalt pavement that equals to 0.6 .

$$
\left\{\begin{array}{l}
\sum \mathbf{F}=m \mathbf{a}=0, \\
\sum \mathbf{F}=\mathbf{F}_{\text {traction }}+\mathbf{F}_{\text {friction }}+\mathbf{F}_{\text {air }}+\mathbf{G} .
\end{array}\right.
$$

For the robot goes for $2.5 \mathrm{~m}$, so that the energy consumption is just equal to the work of traction, as Eq. (20) shown, it is $176.40 \mathrm{~J}$.

$W_{\text {wheeled }}=\mathbf{F}_{\text {traction }} \times \mathbf{s}=70.56 \mathrm{~N} \times 2.5 \mathrm{~m}=176.40 \mathrm{~J}$.

\subsection{The 5-TET Mechanism}

During the straight going in Quadrant I, there are totally 35 executed cylinders, and the overall compressed gas consumption is calculated in Eq. (21):

$$
\begin{aligned}
V & =\pi r^{2} \times \Delta l \times 35 \\
& =3.14 \times\left(8 \times 10^{-3} \mathrm{~m}\right)^{2} \times 0.1 \mathrm{~m} \times 35=0.7034 \mathrm{~L} .
\end{aligned}
$$

For Dry Ice Power Cell, the specific energy of dry ice is $11.63 \mathrm{~W} \cdot \mathrm{h} / \mathrm{kg}$ [25]. $430 \mathrm{~g}$ dry ice could produce $42 \mathrm{~L}$ gas at pressure of $0.42 \mathrm{MPa}$ [28], thus the specific energy of gas carbon dioxide is $4.29 \times 10^{5} \mathrm{~W} \cdot \mathrm{s} / \mathrm{m}^{3}$. According to the energy consumption calculation method proposed by Zhang and Cai [35], the energy consumption of 5-TET that goes along the path of Figure 17(a) actuated with Dry Ice Power Cell is shown in Eq. (22), as $317.38 \mathrm{~J}$.

$$
W=\frac{\alpha_{1} V}{1-\beta}=\frac{4.29 \times 10^{5} \times 0.7034}{1-5 \%}=317.38 \mathrm{~J} .
$$

Where $\alpha_{1}$ represents the specific energy of carbon dioxide, $\beta$ is the gas leakage rate, take an average value as $5 \%$.

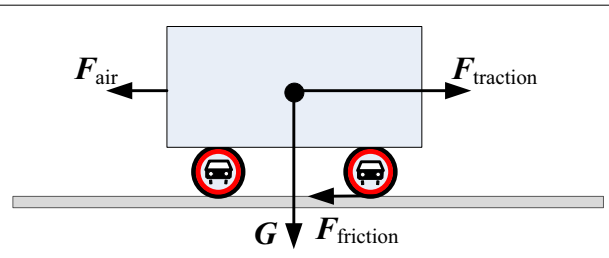

Figure 27 Force diagram of wheeled robot
For compressed air, which uses the air compressor with the power of $550 \mathrm{~W}$ and the output flow of $40 \mathrm{~L} / \mathrm{min}$, the specific energy is obtained in Eq. (23), it is $8.25 \times 10^{5} \mathrm{~W} \cdot \mathrm{s} /$ $\mathrm{m}^{3}$. So that the energy consumption of compressed air is shown in Eq. (24), it is $610.85 \mathrm{~J}$.

$$
\begin{aligned}
& \alpha_{2}=\frac{P_{c}}{Q_{c}}=\frac{550}{0.04 / 60}=8.25 \times 10^{5} \mathrm{Ws} / \mathrm{m}^{3}, \\
& W=\frac{\alpha_{2} V}{1-\beta}=\frac{8.25 \times 10^{5} \times 0.7034}{1-5 \%}=610.85 \mathrm{~J} .
\end{aligned}
$$

Where $\alpha_{2}$ represents the specific energy of compressed air, $P_{\mathrm{c}}$ is the power of air compressor, $Q_{\mathrm{c}}$ is the output flow of air compressor.

From the results of energy consumption analyses, the traditional wheeled robot is the most energy-efficient. And 5-TET supplied by Dry Ice Power Cell consumes less energy than that supplied by compressed air, where the energy-saving rate reaches to approximately $48 \%$.

Moreover, the flexibility and stability are the individual characters of this mechanism. With the frame-like structure, 5-TET can contain the barriers to overcome it easily, as shown in Figure 28. Meanwhile the objective of the optimization is that achieve certain functions by driving minimum number of actuators, which to some extent reduces the energy consumption.

\section{Simulations of Successive Gait and Gripper Function}

To verify the feasibility of 5-TET, the dynamic simulation of locomotion and manipulation were carried out. 5-TET has a total of 18 DOFs, 18 pneumatic cylinders were used to drive the 18 prismatic joints, so that the spherical joints are passive joints.

Figure 29 shows the rolling path of 5-TET along a straight line from $(0 \mathrm{~mm}, 0 \mathrm{~mm})$ to $(1500 \mathrm{~mm}, 2000 \mathrm{~mm})$. During this process, the pneumatic cylinder $\mathrm{BC}$ is locked, and the 17 remaining cylinders are driven alternately

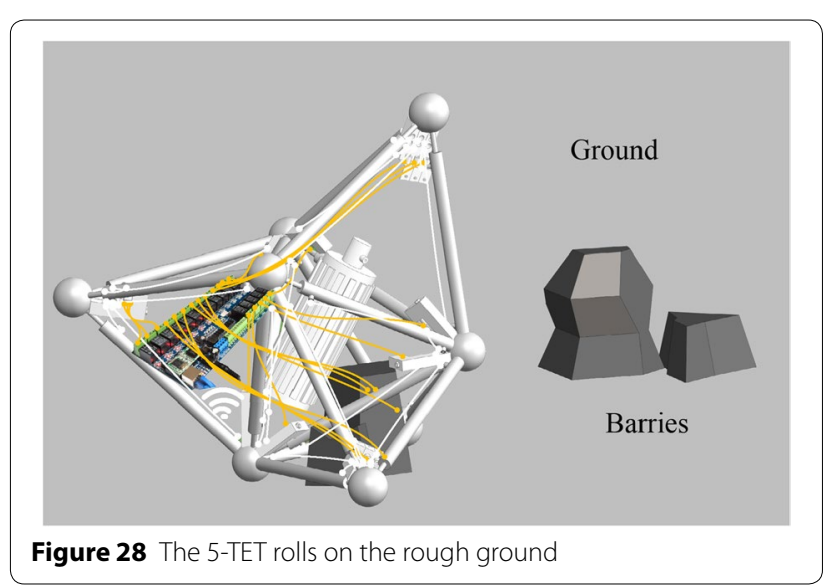




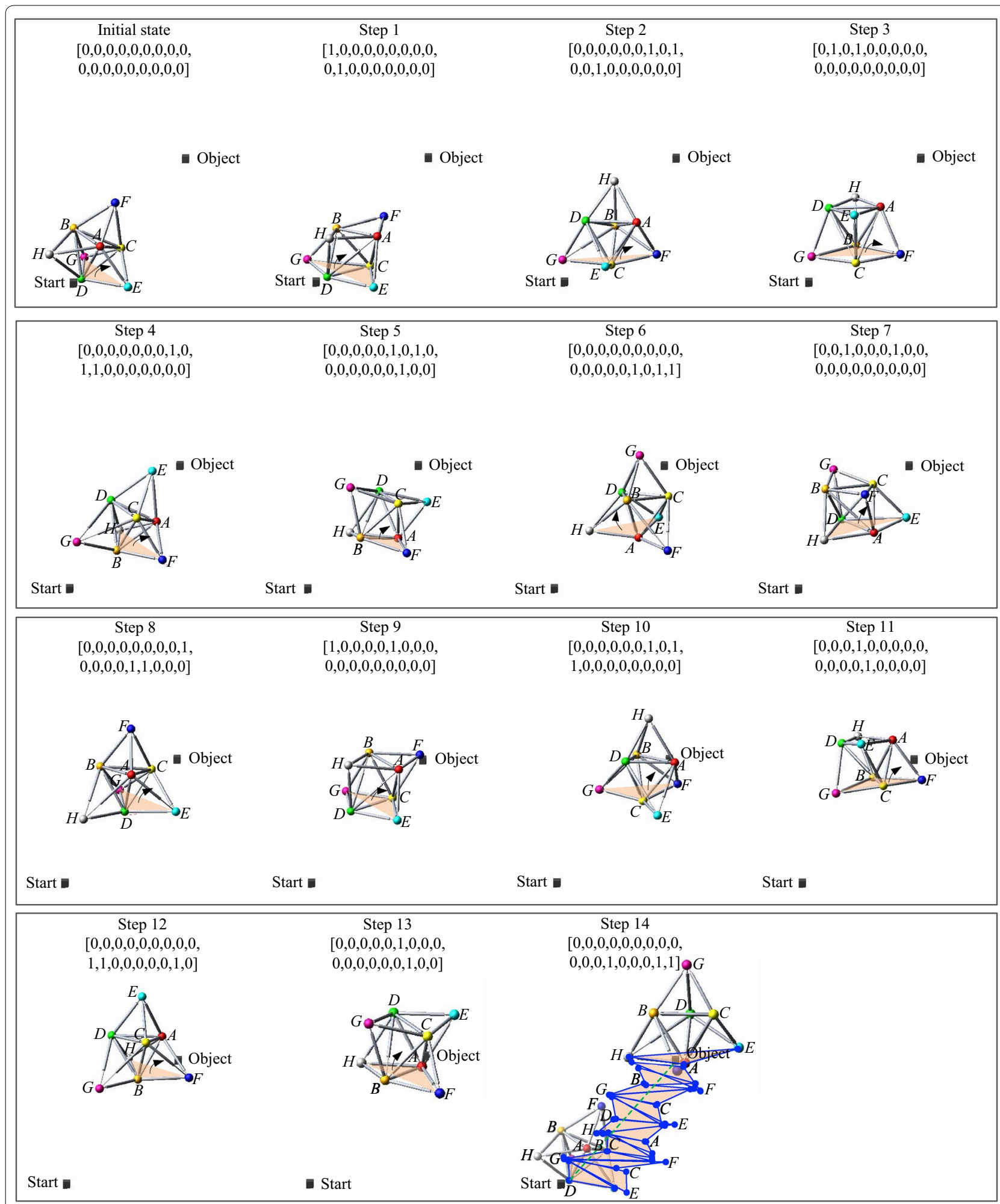

Figure 29 Rolling along a straight line with the target of (1500 mm, $2000 \mathrm{~mm}$ ) 


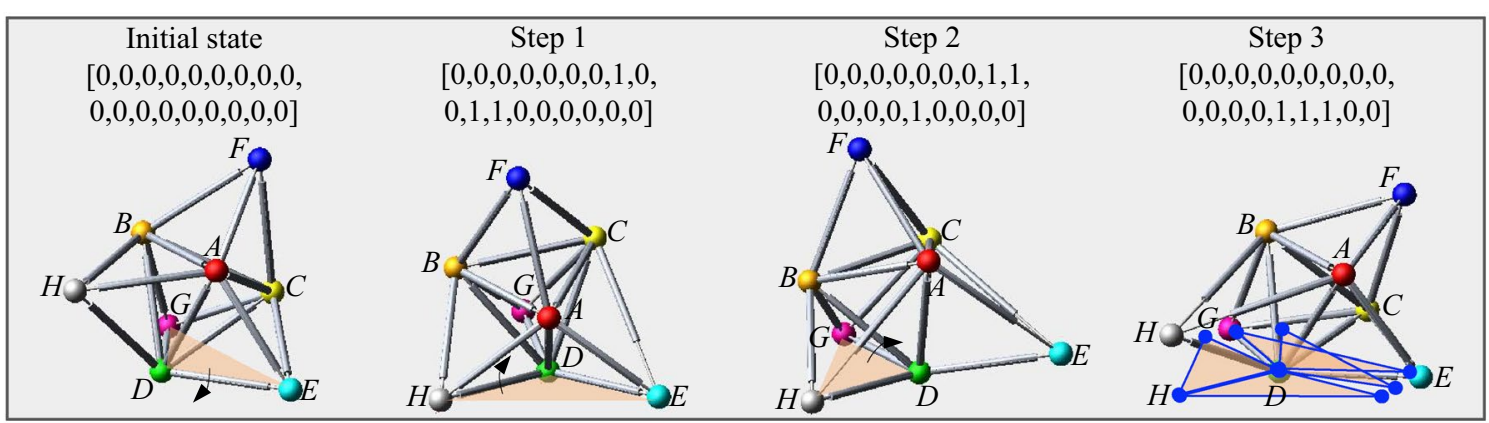

a Normal path

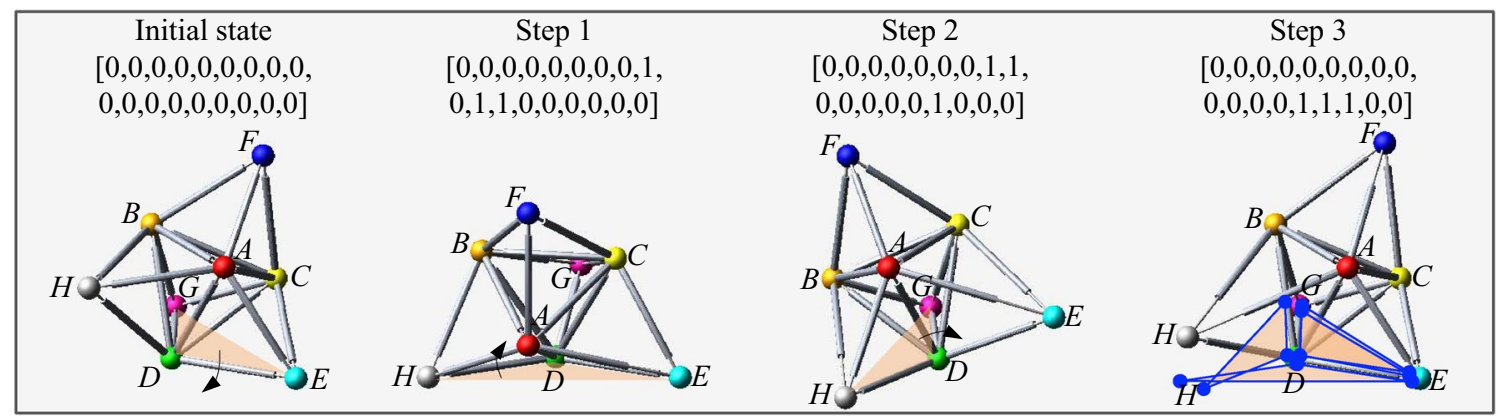

b Position reset turning

Figure 30 Procedure of Case I turning

according to the optimal inputs combinations already obtained (see in Figure 18(b)) to control the rolling directions. Figure 30 shows the turning paths of Case I, the optimal inputs are shown in Figure 20. In Figure 30(a), the 5-TET turned around with three steps, but the support area of the last step and the initial has an overlap. In Figure 30(b), the 5-TET turned around with three steps, then practically reset to its initial position. The turning path of Case II is shown in Figure 31, corresponding to the optimal inputs in Figure 22. Case II has more detailed division about the interval of turning degrees (Additional file 1).

Furthermore, according to the optimal codes in Figure 26(b), the manipulation function of 5-TET was simulated, as Figure 32 shown. The gripper object was set between node $F$ and $H$. Due to the location and size of the object, 5-TET gripped it after four attempts, which correspond to steps 8-11 shown in Figure 26(a).

\section{Conclusions}

(1) An optimization method based on a developed GA is proposed to optimize the motion of binary control 5-TET mechanism. The 5-TET currently realizes its locomotion by executing at most 5 pneumatic cylinders between each two steps, rather than using multiple actuators as before.

(2) A potential manipulation function of the mechanism that operated with two non-support TETs synergistically is presented based on the proposed optimization method.

(3) The energy consumptions of the 5-TET and the traditional wheeled robot are compared. Though the wheeled robot is more energy-saving, the 5-TET using portable supply of Dry Ice Power Cell saves approximately $48 \%$ energy than that supplied by general air compressor.

(4) Dynamic simulations are carried out to validate the proposed algorithm. As a result, the dynamic simulation trajectories consistently match with that of 


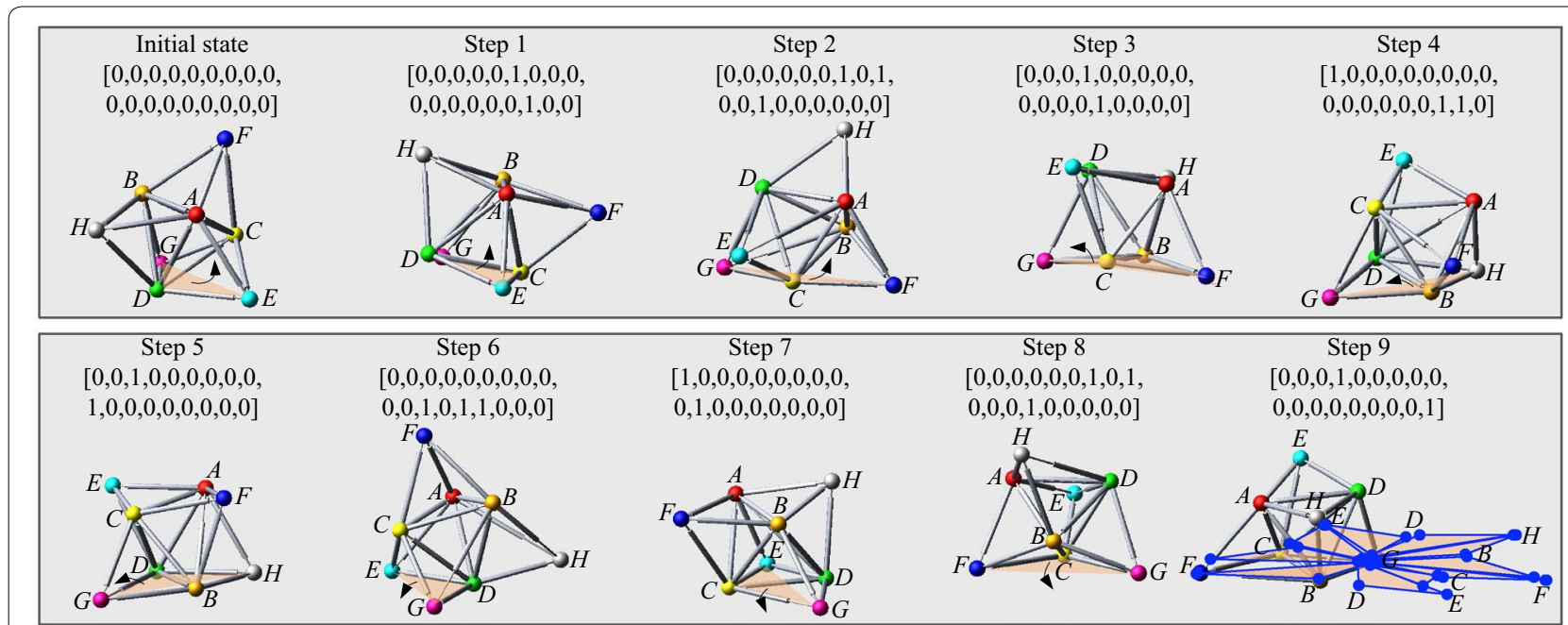

Figure 31 Procedure of Case II turning

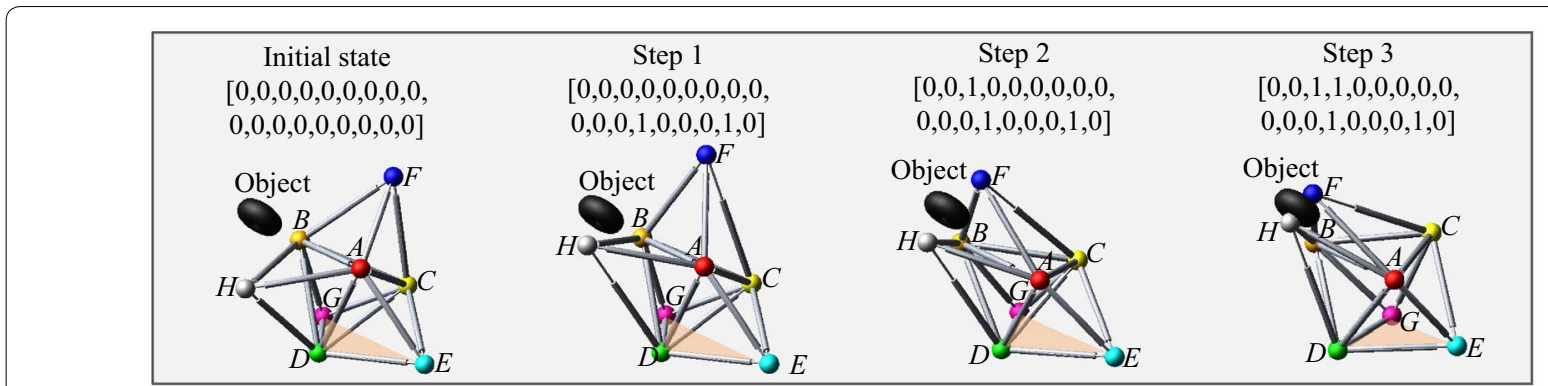

Figure 32 Procedure of gripper manipulation function

the mathematic analyses, which indicates that the optimization is effective both on the locomotion and manipulation planning. Through the multiterrain optimization and the whole system design in the next step, it will prospectively have better performance in more complex environments.

\section{Additional File}

Additional file 1 Simulation animation of successive gait and gripper function.

\section{Authors' information}

Ran Liu born in 1991, is currently a PhD candidate at School of Mechanical, Electronic and Control Engineering, Beijing Jiaotong University, China. She received her bachelor degree from Hebei University of Engineering, China, in 2013. Her research interests include mechanisms and mobile robotics.

Yan-An Yao born in 1972, is currently a professor at School of Mechanical, Electronic and Control Engineering, Beijing Jiaotong University, China. He received his PhD degree from Tianjin University, China, in 1999. His main research interests include mechanisms and mobile robotics.

Wan Ding born in 1987, is currently a postdoctoral fellow at Department of Mechanism Theory and Dynamics of Machines, RWTH Aachen University, Germany. He received his PhD degree in 2015 from Beijing Jiaotong University, China. His research interests include mechanisms and mobile robotics.
Xiao-Ping Liu born in 1970, is currently a professor at School of Mechanical, Electronic and Control Engineering, Beijing Jiaotong University, China. He is a Canada Research Chair Professor at Department of Systems and Computer Engineering, Carleton University, Canada. He received his PhD degree from the University of Alberta, Canada. His research interests include intelligent systems and robotics.

\section{Author details}

${ }^{1}$ School of Mechanical, Electronic and Control Engineering, Beijing Jiaotong University, Beijing 100044, China. ${ }^{2}$ Department of Mechanism Theory and Dynamics of Machines, RWTH Aachen University, 52072 Aachen, Germany. ${ }^{3}$ Department of Systems and Computer Engineering, Carleton University, Ottawa, ON K1S 5B6, Canada.

\section{Authors' contributions}

RL carried out gait optimization studies, participated in the dynamic analysis and drafted the manuscript. YY completed the mechanism design and modeling, participated in the gripper function planning. WD presented the kinematic analysis, participated in the dynamic simulation. XL participated in the improved genetic algorithm. All authors read and approved the final manuscript.

\section{Acknowledgements}

Supported by National Science-Technology Support Plan Projects of China (Grant No. 2015BAK04B00), and 2015 Sino-German Postdoc Scholarship Program (Grant No. 57165010). 


\section{Competing interests}

The authors declare that they have no competing interests.

\section{Ethics approval and consent to participate}

Not applicable.

\section{Publisher's Note}

Springer Nature remains neutral with regard to jurisdictional claims in published maps and institutional affiliations.

Received: 4 April 2016 Accepted: 15 January 2018

Published online: 27 February 2018

\section{References}

1. L Bruzzone, G Quaglia. Locomotion systems for ground mobile robots in unstructured environments. Mechanical Sciences, 2012, 3(2): 49-62.

2. J G Liu, Y C Wang, B Li, et al. Current research, key performances and future development of search and rescue robots. Journal of Mechanical Engineering, 2006, 42(12): 1-12 (in Chinese).

3. J Sastra, S Chitta, M Yim. Dynamic rolling for a modular loop robot. The International Journal of Robotics Research, 2009, 28(6): 758-773.

4. K Cook, J Swett. 13th annual celebration for undergraduate research and creative performace: design and simulation of tetrahedral robotics. Michigan: Hope College, 2014 [2017-02-04]. http://digitalcommons.hope.edu/ curcp_13/64.

5. G J Hamlin, A C Sanderson. Tetrobot: a modular system for hyper-redundant parallel robotics. Proceedings of the IEEE International Conference on Robotics and Automation, Nagoya, Japan, May 21-27, 1995: 154-159.

6. X B Chen, F Gao, C K Qi, et al. Gait planning for a quadruped robot with one faulty actuator. Chinese Journal of Mechanical Engineering, 2015, 28(1) 11-19.

7. G S Chirikjian, JW Burdick. The kinematics of hyper-redundant robot locomotion. IEEE Transactions on Robotics and Automation, 1995, 11(6): 781-793.

8. K Cook, M Abrahantes. Gait design for a tetrahedral worm. Proceedings of the IEEE International Conference on Electro-Information Technology, Grand Forks, USA, May 19-21, 2016: 0621-0626.

9. J Friesen, A Pogue, T Bewley, et al. DuCTT: a tensegrity robot for exploring duct systems. Proceedings of the IEEE International Conference on Robotics and Automation, Hong Kong, China, May 31-June 7, 2014: 4222-4228.

10. J M Friesen, P Glick, M Fanton, et al. The second generation prototype of a duct climbing tensegrity robot, DuCTTV2. Proceedings of the IEEE International Conference on Robotics and Automation, Stockholm, Sweden, May 16-21, 2016: 2123-2128.

11. DT Margineanu, E C Lovasz, V Ciupe, et al. Tetrahedral mechanism crawling on a slope. Proceedings of the 14th IFTOMM World Congress, Taipei, Taiwan, China, October 25-30, 2015: 705-712.

12. C H Yu, R Nagpal. Self-adapting modular robotics: a generalized distributed consensus framework. Proceedings of the IEEE International Conference on Robotics and Automation, Kobe, Japan, May 12-17, 2009: 1881-1888.

13. BT Mirletz, P Bhandal, R D Adams, et al. Goal-directed CPG-based control for tensegrity spines with many degrees of freedom traversing irregular terrain. Soft Robotics, 2015, 2(4): 165-176.

14. STabandeh, W M Melek, C M Clark. An adaptive niching genetic algorithm approach for generating multiple solutions of serial manipulator inverse kinematics with applications to modular robots. Robotica, 2010, 28(4): 493-507.

15. J K Parker, A R Khoogar, D E Goldberg. Inverse kinematics of redundant robots using genetic algorithms. Proceedings of the IEEE International Conference on Robotics and Automation, Scottsdale, USA, May 14-19, 1989: $271-276$
16. L G Zhang, S S Bi, Y R Cai. Design and motion analysis of tetrahedral rolling robot. Proceedings of the IEEE/RSJ International Conference on Intelligent Robots and Systems, Taipei, Taiwan, China, October 18-22, 2010: 502-507.

17. J Swett, M Abrahantes. Distributed control system implementation for tetrahedral walker robots. Proceedings of the IEEE International Conference on Electro/Information Technology, Milwaukee, USA, June 5-7, 2014: 231-235.

18. W Ding, J XWu, Y A Yao. Three-dimensional construction and omni-directional rolling analysis of a novel frame-like lattice modular robot. Chinese Journal of Mechanical Engineering, 2015, 28(4): 691-701.

19. $X L$ Ding, $Y$ Zhang, $K X$ U. Wheel-legged hexapod robots: a multifunctional mobile manipulating platform. Chinese Journal of Mechanical Engineering, 2017, 30(1): 3-6.

20. R Liu, Y A Yao. A rolling triangular-bipyramid robot covering bennett linkage. Proceedings of ASIAN MMS 2016 and CCMMS 2016, Guangzhou, China, December 15-17, 2016: 415-427.

21. W Ding, S C Kim, Y A Yao. A pneumatic cylinder driving polyhedron mobile mechanism. Frontiers of Mechanical Engineering, 2012, 7(1): 55-65.

22. V A Sujan, M D Lichter, S Dubowsky. Lightweight hyper-redundant binary elements for planetary exploration robots. Proceedings of the IEEE/ASME International Conference on Advanced Intelligent Mechatronics, Como, Italy, July 8-12, 2001: 1273-1278.

23. V A Sujan, S Dubowsky. Design of a lightweight hyper-redundant deployable binary manipulator. Journal of Mechanical Design, 2004, 126(1): 29-39.

24. J Suthakorn. Binary hyper-redundant robotic manipulator concept. IEEE Region 10 Conference, Chiang Mai, Thailand, November 21-24, 2004: 625-628.

25. M A Erdmann, MT Mason. An exploration of sensorless manipulation. IEEE Journal on Robotics and Automation, 1988, 4(4): 369-379.

26. C Giuseppe. Experimental characterization of a binary actuated parallel manipulator. Chinese Journal of Mechanical Engineering, 2016, 29(3): 445-453.

27. J H Han, T Noritsugu. Development of a miniature air compressor driven with a linear electromagnetic actuator. 6th International Conference on Fluid Power Transmission and Control, Hangzhou, China, April 5-8, 2005: 377-380.

28. J A Riofrio, E J Barth. A free piston compressor as a pneumatic mobile robot power supply: design, characterization and experimental operation. International Journal of Fluid Power, 2007, 8(1): 17-28.

29. HWu, A Kitagawa, H Tsukagoshi, et al. Development and testing of a novel portable pneumatic power source using phase transition at the triple point. Proceedings of the Institution of Mechanical Engineers, Part C: Journal of Mechanical Engineering Science, 2009, 223(6): 1425-1432.

30. C A Klein, T A Miklos. Spatial robotic isotropy. The International Journal of Robotics Research, 1991, 10(4): 426-437.

31. H B Qu, Y F Fang, S Guo. A new method for isotropic analysis of limited DOF parallel manipulators with terminal constraints. Robotica, 2011, 29(4): 563-569.

32. F H F Leung, H K Lam, S H Ling, et al. Tuning of the structure and parameters of a neural network using an improved genetic algorithm. IEEE Transactions on Neural Networks, 2003, 14(1): 79-88

33. A T Ismail, A Sheta, A W Mohammed. A mobile robot path planning using genetic algorithm in static environment. Journal of Computer Science, 2008, 4(4): 341-344

34. H Terasaki, T Hasegawa. Motion planning of intelligent manipulation by a parallel two-fingered gripper equipped with a simple rotating mechanism. IEEE Transactions on Robotics and Automation, 1998, 14(2): 207-219.

35. Y M Zhang, M L Cai. Energy consumption analysis for pneumatic actuator and electric actuator. Journal of Beijing University of Aeronautics and Astronautics, 2010, 36(5): 560-563 (in Chinese). 